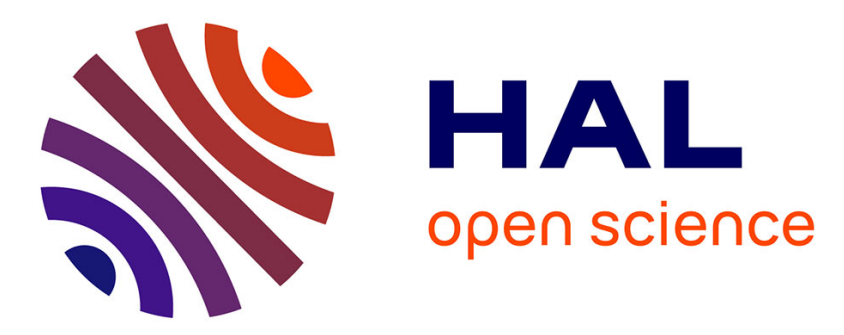

\title{
Non-measurability properties of interpolation vector spaces
}

Friedrich Wehrung

\section{To cite this version:}

Friedrich Wehrung. Non-measurability properties of interpolation vector spaces. Israel Journal of Mathematics, 1998, 103 (1), pp.177-206. 10.1007/BF02762273 . hal-00004065

\section{HAL Id: hal-00004065 \\ https://hal.science/hal-00004065}

Submitted on 25 Jan 2005

HAL is a multi-disciplinary open access archive for the deposit and dissemination of scientific research documents, whether they are published or not. The documents may come from teaching and research institutions in France or abroad, or from public or private research centers.
L'archive ouverte pluridisciplinaire HAL, est destinée au dépôt et à la diffusion de documents scientifiques de niveau recherche, publiés ou non, émanant des établissements d'enseignement et de recherche français ou étrangers, des laboratoires publics ou privés. 


\title{
NON-MEASURABILITY PROPERTIES OF INTERPOLATION VECTOR SPACES.
}

\author{
Friedrich WEHRUNG \\ Université de Caen \\ Département de Mathématiques \\ 14032 CAEN CEDEX, FRANCE
}

\begin{abstract}
It is known that every dimension group with order-unit of size at most $\aleph_{1}$ is isomorphic to $K_{0}(R)$ for some locally matricial ring $R$ (in particular, $R$ is von Neumann regular); similarly, every conical refinement monoid with order-unit of size at most $\aleph_{1}$ is the image of a V-measure in Dobbertin's sense, the corresponding problems for larger cardinalities being open. We settle these problems here, by showing a general functorial procedure to construct ordered vector spaces with interpolation and order-unit $E$ of cardinality $\aleph_{2}$ (or whatever larger) with strong non-measurability properties. These properties yield in particular that $E^{+}$is not measurable in Dobbertin's sense, or that $E$ is not isomorphic to the $K_{0}$ of any von Neumann regular ring, or that the maximal semilattice quotient of $E^{+}$is not the range of any weak distributive homomorphism (in E.T. Schmidt's sense) on any distributive lattice, thus respectively solving problems of Dobbertin, Goodearl and Schmidt.
\end{abstract}

\section{$\S 0$. Introduction.}

Let $M$ be a commutative monoid and let $L$ be a lattice with 0 . Say that a map $\mu: L \rightarrow M$ is a measure when $\mu(0)=0$ and $\mu(a \vee b)=\mu(a)+\mu(b)$ whenever $a \wedge b=0$; furthermore, if $L$ has a largest element 1 and if we have fixed a distinguished element $e$ of $M$, say that $\mu$ is normalized when $\mu(1)=e$. In his paper [5], H. Dobbertin considers Vaught measures, or $\mathbf{V}$-measures, i.e., measures $\mu: B \rightarrow M$ where $B$ is a Boolean algebra, satisfying the following $\mathbf{V}$-condition: if $\mu(c)=\alpha+\beta$, then there are $a$ and $b$ such that $a \wedge b=0$, $a \vee b=c$ and $\mu(a)=\alpha, \mu(b)=\beta$. For example, the Lebesgue measure on the Borel algebra of $[0,1]$ is a $\mathrm{V}$-measure. Another example of $\mathrm{V}$-measure is obtained by taking a countable Boolean algebra $B$ and the commutative monoid BA of all isomorphism types of countable Boolean algebras, and defining $\mu: B \rightarrow$ BA by $\mu(a)=$ isomorphism type of $B \mid a$ where $B \mid a=\{x \in B: x \leq a\}$. At the present time, the deepest result about the latter example is probably J. Ketonen's difficult result [17] that every countable commutative semigroup can be embedded into BA. If $\mu: B \rightarrow M$ is a $\mathrm{V}$-measure, then $M$ is conical,

1991 Mathematics subject classification: 06F20, 06A12, 08C99, 06C20, 28B10, 03E05, 19A49, $19 \mathrm{~K} 14$.

Key words and phrases: Kuratowski's Theorem; refinement monoids; interpolation; ordered vector spaces; measures; semilattices; lattices; weak distributive homomorphisms. 
i.e., it satisfies the axiom $(\forall x, y)(x+y=0 \Rightarrow x=y=0)$. As in [26], say that a cone is a conical commutative monoid. Another property satisfied by ranges of $\mathrm{V}$-measures is that if $\mu: B \rightarrow M$ is a $\mathrm{V}$-measure, then the following (Riesz) refinement property

$$
\left(\forall_{i<2} a_{i}, b_{i}\right)\left[a_{0}+a_{1}=b_{0}+b_{1} \Rightarrow\left(\exists_{i, j<2} c_{i j}\right)(\forall i<2)\left(a_{i}=c_{i 0}+c_{i 1} \text { and } b_{i}=c_{0 i}+c_{1 i}\right)\right]
$$

is satisfied by all elements of $M$ satisfying $a_{0}+a_{1}=b_{0}+b_{1} \leq e$. Consequently, we will mainly focus attention on refinement cones, i.e., cones satisfying the refinement property.

Thus to be the range of a $\mathrm{V}$-measure is quite restrictive, and in fact, countable Boolean algebras $\left(V_{-}\right)$measured by the same pointed monoid are isomorphic (see for example [19, 8.4]); this is easily seen to fail in the uncountable case. On the existence side, it can be shown [5, Theorem 3.4] that if $M$ is a refinement cone with cardinality (or even sum rank) at most $\aleph_{1}$, then $M$ is measurable in the sense that for every $e \in M$, there exists a normalized $\mathrm{V}$-measure with target $(M, e)$. It is also proved among other things in [7] that distributive lattices with 0 , abelian groups with an extra zero element adjoined and positive cones of abelian $\ell$-groups are measurable. For refinement cones of arbitrary cardinality, the measurability problem was left open.

We solve this problem here (negatively), by constructing an example of an ordered vector space over the rationals with interpolation and order-unit of cardinality $\aleph_{2}$ whose positive cone is not measurable, and in fact fails a seemingly much weaker property than measurability, the amalgamation property proved by Dobbertin to be true in the case of $\aleph_{1}$ finite Boolean algebras in [7, Theorem 6] (but which fails for $\aleph_{2}$ two-atoms Boolean algebras by our example). In fact, it turns out that the negative property satisfied by our example is so strong that it also solves (negatively) two other known open problems, one of them due to K.R. Goodearl [10, Problem 30], the other one due to E.T. Schmidt [21, Problem 9]. The first of these two problems is to know whether every dimension group with order-unit is isomorphic to the $K_{0}$ of a direct limit of matricial algebras, or even to the $K_{0}$ of a unit-regular ring [12]: up to now, the state of knowledge was that the answer was "yes" for dimension groups of cardinality at most $\aleph_{1}[\mathbf{8}, \mathbf{9}, \mathbf{1 3}]$; thus this $\aleph_{1}$ bound is optimal. The second of these two problems is to know whether every distributive semilattice with zero is the image of a generalized Boolean algebra by a "weak distributive homomorphism" (Definition 2.13); again, the answer was known to be positive for semilattices of size at most $\aleph_{1}[\mathbf{1 6}, \mathbf{2 3}]$.

We shall now outline the organization of the paper. In Section 1, we give a general procedure of embedding any arbitrary ordered vector space (over a given totally ordered field) 
into an interpolation vector space. Although there already exist many such embedding results (see for example [15, Theorem 1] and [5, Theorem 5.1] for commutative monoids, or [25] for positively preordered commutative monoids, or [26] for cones or partially ordered abelian groups), it is very important for our purposes to make this procedure uniform. This uniformity is best described by Propositions 1.16 and 1.17, which show that the embedding procedure is given by a functor $\mathbf{J}_{K}$, which in addition preserves direct limits; roughly speaking, if $E$ is a partially ordered $K$-vector space, then $\mathbf{J}_{K}(E)$ is a kind of "interpolation vector space hull" of $E$. The embedding functor $\mathbf{J}_{K}$ is obtained by an infinite countable iteration of a "one-step" embedding procedure, also described by a direct limits preserving functor $\mathbf{I}_{K}$. Roughly speaking, if $E$ is an ordered vector space, the space $\mathbf{I}_{K}(E)$ is freely generated over $E$ by (two-by-two) cuts on $E$, the only relations being those saying that the element corresponding to the cut $\left\langle a_{0}, a_{1}, b_{0}, b_{1}\right\rangle$ lies between $a_{0}, a_{1}$ on one side and $b_{0}, b_{1}$ on the other side; it turns out that the ordering on $\mathbf{I}_{K}(E)$ can then be described in a "computable" way, using considerations of convexity. Due to the resulting syntactical complexity of the elements of $\mathbf{J}_{K}(E)$, we need the notion of interpolator (Definition 1.18) which will allow us, via Propositions 1.21 and 1.22, to restrict the problems of Section 2 to a "computable" part of $\mathbf{J}_{K}(E)$ - in fact, the first level $\mathbf{I}_{K}(E)$.

Next, in Section 2, we apply the embedding functor $\mathbf{J}_{K}$ to a certain very simple explicitly defined ordered vector space $\mathbf{E}_{K}(\Omega)$ where $\Omega$ is an arbitrary set, to obtain a space which we shall denote by $\mathbf{F}_{K}(\Omega)$. The main result (Theorem 2.8) shows that if $\Omega$ has large enough cardinality, then the space $\mathbf{F}_{K}(\Omega)$ has strong non-measurability properties. The key fact for this is a simple infinite combinatorial statement going back to C. Kuratowski [18], showing that the appropriate lower bound for the cardinality of $\Omega$ is $\aleph_{2}$ (this bound is optimal, for example by Dobbertin's results [5]).

To conclude Section 2, we will prove a version of Theorem 2.8 for the maximal semilattice quotient of $\mathbf{F}_{K}(\Omega)^{+}$(Theorem 2.15): it turns out that the latter is a distributive semilattice of cardinality $\aleph_{2}$, and that it even fails to be the range of a weak distributive homomorphism (Definition 2.13) on a distributive lattice as defined by E.T. Schmidt $[\mathbf{2 1}, \mathbf{2 2}]$ (thus, it is not the range of any $\mathrm{V}$-measure on a Boolean algebra). This proves that Schmidt's approach via distributive homomorphisms on generalized Boolean algebras to solve positively the "Congruence Lattice Problem" (see for example [23] for a survey) - i.e., whether any distributive algebraic lattice is the congruence lattice of a lattice is doomed for general distributive semilattices of size $\geq \aleph_{2}$. Note that this time, the corresponding proof cannot be extended to give a negative result about the K-theory of von Neumann regular rings since it makes use in an essential way of the distributivity of 
the domain of the measure. Let us point out that $\mathrm{H}$. Dobbertin informed us in a private communication that he had independently constructed a distributive semilattice of cardinality $2^{2^{\aleph_{0}}}$ which is not the range of any weak distributive homomorphism on a distributive lattice.

We will mostly follow standard set-theoretical notation and terminology. Thus the set of all natural numbers, which is also the first limit ordinal, will be denoted by $\omega$; in particular, for every natural number $n$, we have $n=\{0,1, \ldots, n-1\}$. More generally, every ordinal number is the set of all its predecessors. For every ordinal $\xi, \omega_{\xi}$ will denote the $\xi^{\text {th }}$ infinite initial ordinal, as usual denoted by $\aleph_{\xi}$ when viewed as a cardinal number. For all sets $X$ and $Y, X^{Y}$ denotes the set of all maps from $Y$ to $X$. If $f: X \rightarrow Y$ is a function, then for all $Z \subseteq X, f[Z]$ denotes the image of $Z$ under $f$ while for all $Z \subseteq Y, f^{-1}[Z]$ denotes the inverse image of $Z$ under $f$. If $M$ is a monoid and $X$ is a set, we will denote by $M^{(X)}$ the submonoid of $M^{X}$ consisting of those maps $f: X \rightarrow M$ such that $f^{-1}[M \backslash\{0\}]$ is finite. If $S$ is a set and $n \in \omega$, we will denote by $[S]^{n}$ the set of all $n$-element subsets of $S$, and we will put $[S]^{<\omega}=\bigcup_{n \in \omega}[S]^{n}$.

If $(P, \leq)$ is an ordered set and if $\left\langle a_{1}, \ldots, a_{m}\right\rangle$ and $\left\langle b_{1}, \ldots, b_{n}\right\rangle$ are finite sequences of elements of $P$, we will abbreviate by $a_{1}, \ldots, a_{m} \leq b_{1}, \ldots, b_{n}$ the statement $(\forall i, j)\left(a_{i} \leq b_{j}\right)$. The interpolation property is the statement

$$
\left(\forall a_{0}, a_{1}, b_{0}, b_{1}\right)\left(a_{0}, a_{1} \leq b_{0}, b_{1} \Rightarrow(\exists x)\left(a_{0}, a_{1} \leq x \leq b_{0}, b_{1}\right)\right) .
$$

If $G$ is an abelian lattice-ordered group and $x$ and $y$ are elements of $G$, we will abbreviate $x \backslash y=x-x \wedge y$. A semilattice $[\mathbf{1 4}]$ is a commutative semigroup where every element is idempotent; when equipped with the partial ordering $\leq$ defined by $x \leq y$ if and only if $x+y=x$ (resp. $x+y=y$ ), it is said to be a meet-semilattice (resp. join-semilattice), in which case the binary operation + becomes the infimum (resp. the supremum) with respect to $\leq$. A semilattice $S$ is distributive [14] when it satisfies the Riesz decomposition property, i.e., the axiom

$$
(\forall a, b, c)(c \leq a+b \Rightarrow(\exists x, y)(x \leq a \text { and } y \leq b \text { and } c=x+y)) ;
$$

it is an easy fact that $S$ satisfies the refinement property if and only if $S$ is distributive.

Finally, if $\mathbf{C}$ and $\mathbf{D}$ are categories, a functor $\mathbf{F}: \mathbf{C} \rightarrow \mathbf{D}$ is as usual said to preserve direct limits when for every direct system (indexed by a directed ordered set $I$ ) $\left(E_{i}, f_{i j}\right)_{i \leq j}$ in $I$ in $\mathbf{C}$ admitting as direct limit $E$ with limiting morphisms $f_{i}: E_{i} \rightarrow E$ (all $i \in I)$, the image direct system $\left(\mathbf{F}\left(E_{i}\right), \mathbf{F}\left(f_{i j}\right)\right)_{i \leq j}$ in $I$ admits $\mathbf{F}(E)$ as direct limit with limiting morphisms $\mathbf{F}\left(f_{i}\right)$ (all $i \in I$ ). 


\section{$\S 1$. Construction of the functors $\mathbf{I}_{K}$ and $\mathbf{J}_{K}$.}

Throughout this section, we will fix a totally ordered field $K$. We will put $[0,1]=\{x \in$ $K: 0 \leq x \leq 1\}$. If $E$ is a vector space over $K$ and $a$ and $b$ are elements of $E$, we define the segment $[a, b]=\{(1-\lambda) a+\lambda b: \lambda \in[0,1]\}$. A subset $X$ of $E$ is convex when for all elements $a$ and $b$ of $X$, the segment $[a, b]$ is contained in $X$. We will work in the category of (partially) ordered K-vector spaces; therefore, from now on, by "positive homomorphism", we will always mean a homomorphism of ordered $K$-vector spaces, i.e., a positive $K$-linear map. For all ordered $K$-vector spaces $E$ and $F$, we will denote by $\operatorname{Hom}_{K}^{+}(E, F)$ the set of all positive homomorphisms from $E$ to $F$.

For every ordered $K$-vector space $E$, we will put

$$
\mathrm{C}(E)=\left\{p \in E^{4}: p(0), p(1) \leq p(2), p(3)\right\} .
$$

For every element $p$ of $\mathrm{C}(E)$, we will put $p^{-}=[p(0), p(1)]$ and $p^{+}=[p(2), p(3)]$. An interpolant of $p$ in $E$ is an element $c$ of $E$ such that $p(0), p(1) \leq c \leq p(2), p(3)$. We will say that $p$ is trivial when $\{p(0), p(1)\} \cap\{p(2), p(3)\} \neq \emptyset$. The space $E$ is an interpolation vector space when every element of $\mathrm{C}(E)$ has an interpolant in $E$.

From 1.1 to 1.10 , we will fix an ordered $K$-vector space $E$.

Lemma 1.1. An element $p$ of $\mathrm{C}(E)$ is trivial if and only if $p^{+} \cap p^{-} \neq \emptyset$.

Proof. Suppose that $p$ is non trivial and let $x \in p^{-}, y \in p^{+}$. By definition, there are elements $\alpha$ and $\beta$ of $[0,1]$ such that $x=(1-\alpha) p(0)+\alpha p(1)$ and $y=(1-\beta) p(2)+\beta p(3)$. Since $p(0), p(1)<p(2)$, we have $x<p(2)$; similarly, $x<p(3)$; whence $x<y$. Thus $p^{+} \cap p^{-}=\emptyset$. The converse is trivial.

In the sequel, we will make heavy use of the vector space $K^{(\mathrm{C}(E))}$; we shall of course identify every element $p \in \mathrm{C}(E)$ with the corresponding element of the canonical basis of $K^{(\mathrm{C}(E))}$. Next, put

$$
\Sigma(E)=\prod_{p \in C(E)} p^{+} \quad \text { and } \quad \Pi(E)=\prod_{p \in C(E)} p^{-}
$$

and define a binary relation $\ll$ on $\left(K^{+}\right)^{(\mathrm{C}(E))}$ by

$$
x \ll y \Longleftrightarrow(\exists u \in \Sigma(E))(\exists v \in \Pi(E))\left(\sum_{p \in \mathrm{C}(E)} x(p) u(p) \leq \sum_{p \in \mathrm{C}(E)} y(p) v(p)\right)
$$

Note that we only need to choose the elements $u(p)$ and $v(p)$ for those $p$ such that $x(p) \neq 0$ or $y(p) \neq 0$. Note also that it is possible to have $x \ll x$ (for example, $0 \ll 0$ ). 
Lemma 1.2. Let $x=\sum_{i<n} x_{i} p_{i}$ and $y=\sum_{i<n} y_{i} p_{i}$ where $n \in \omega, x_{i}, y_{i} \in K^{+}$and $p_{i} \in \mathrm{C}(E)$ (all $\left.i<n\right)$. Then $x \ll y$ if and only if there exist finite sequences $\left(u_{i}\right)_{i<n}$ and $\left(v_{i}\right)_{i<n}$ such that for all $i<n, u_{i} \in p_{i}^{+}$and $v_{i} \in p_{i}^{-}$and $\sum_{i<n} x_{i} u_{i} \leq \sum_{i<n} y_{i} v_{i}$.

Proof. For all $p \in \mathrm{C}(E)$, put $Z_{p}=\left\{i<n: p_{i}=p\right\}$. Note that for all $p \in \mathrm{C}(E)$, we have $x(p)=\sum_{i \in Z_{p}} x_{i}$ and $y(p)=\sum_{i \in Z_{p}} y_{i}$.

Suppose first that the given condition is realized. For all $p \in \mathrm{C}(E)$, there exist, since both $p^{+}$and $p^{-}$are convex, elements $u(p) \in p^{+}$and $v(p) \in p^{-}$such that

$$
\sum_{i \in Z_{p}} x_{i} u_{i}=\left(\sum_{i \in Z_{p}} x_{i}\right) u(p) \quad \text { and } \quad \sum_{i \in Z_{p}} y_{i} v_{i}=\left(\sum_{i \in Z_{p}} y_{i}\right) v(p)
$$

whence

$$
\sum_{i \in Z_{p}} x_{i} u_{i}=x(p) u(p) \quad \text { and } \quad \sum_{i \in Z_{p}} y_{i} v_{i}=y(p) v(p)
$$

Therefore, $\sum_{p} x(p) u(p)=\sum_{i<n} x_{i} u_{i} \leq \sum_{i<n} y_{i} v_{i}=\sum_{p} y(p) v(p)$, whence $x \ll y$.

Conversely, suppose that $x \ll y$, and let $u \in \Sigma(E)$ and $v \in \Pi(E)$ witness it. For all $i<n$, put $u_{i}=u\left(p_{i}\right)$ and $v_{i}=v\left(p_{i}\right)$; thus $u_{i} \in p_{i}^{+}$and $v_{i} \in p_{i}^{-}$. Moreover,

$$
\begin{aligned}
\sum_{i<n} x_{i} u_{i} & =\sum_{p}\left(\sum_{i \in Z_{p}} x_{i}\right) u(p) \\
& =\sum_{p} x(p) u(p) \\
& \leq \sum_{p} y(p) v(p) \\
& =\sum_{p}\left(\sum_{i \in Z_{p}} y_{i}\right) v(p) \\
& =\sum_{i<n} y_{i} v_{i}
\end{aligned}
$$

whence the announced condition is satisfied.

Lemma 1.3. Let $x, y$ and $z$ be elements of $\left(K^{+}\right)^{(\mathrm{C}(E))}$ such that $0 \leq z \leq x, y$. Then $x \ll y$ implies $x-z \ll y-z$.

Proof. By definition, there exist $u \in \Sigma(E)$ and $v \in \Pi(E)$ such that $\sum_{p} x(p) u(p) \leq$ 
$\sum_{p} y(p) v(p)$. It follows that

$$
\begin{aligned}
\sum_{p}(x(p)-z(p)) u(p) & =\sum_{p} x(p) u(p)-\sum_{p} z(p) u(p) \\
& \leq \sum_{p} y(p) v(p)-\sum_{p} z(p) v(p) \quad(\text { we use } z(p) \geq 0 \text { and } u(p) \geq v(p)) \\
& =\sum_{p}(y(p)-z(p)) v(p),
\end{aligned}
$$

whence $x-z \ll y-z$.

Now, define a binary relation $\sqsubseteq$ on $K^{(\mathrm{C}(E))}$ by putting

$$
x \sqsubseteq y \Longleftrightarrow(\exists z \leq x, y)(x-z \ll y-z)
$$

where $\leq$ denotes the componentwise ordering of $K^{(\mathrm{C}(E))}$.

Lemma 1.4. For all elements $x$ and $y$ of $K^{(\mathrm{C}(E))}, x \sqsubseteq y$ if and only if $x \backslash y \ll y \backslash x$.

Here, $x \backslash y$ is of course computed relatively to the natural structure of lattice-ordered group on $K^{(\mathrm{C}(E))}$.

Proof. If $x \sqsubseteq y$, then, by definition, there exists $z \leq x, y$ such that $x-z \ll y-z$. Put $t=x \wedge y-z$. Then $0 \leq t \leq x-z, y-z$, thus, by Lemma 1.3, $x-z-t \ll y-z-t$, i.e., $x-x \wedge y \ll y-x \wedge y$. The converse is trivial.

The proof of the following lemma is easy:

Lemma 1.5. For all $x, y, z$ in $K^{(\mathrm{C}(E))}$ and all $\lambda \in K^{+}, x \sqsubseteq y$ implies $x+z \sqsubseteq y+z$ and $\lambda x \sqsubseteq \lambda y$.

Lemma 1.6. The relation $\sqsubseteq$ is reflexive.

Proof. For all $x \in K^{(\mathrm{C}(E))}$, we have $x-x \ll x-x$.

Lemma 1.7. The relation $\sqsubseteq$ is transitive.

Proof. Let $x \sqsubseteq y$ and $y \sqsubseteq z$. By definition, there exist elements $r$ and $s$ of $K^{(\mathrm{C}(E))}$ such that $r \leq x, y$ and $s \leq y, z$, and $x-r \ll y-r$ and $y-s \ll z-s$. Thus by definition, there are elements $u^{\prime}, u^{\prime \prime} \in \Sigma(E)$ and $v^{\prime}, v^{\prime \prime} \in \Pi(E)$ such that

$$
\sum_{p}(x(p)-r(p)) u^{\prime}(p) \leq \sum_{p}(y(p)-r(p)) v^{\prime}(p)
$$

and

$$
\sum_{p}(y(p)-s(p)) u^{\prime \prime}(p) \leq \sum_{p}(z(p)-s(p)) v^{\prime \prime}(p) .
$$


But for all $p \in \mathrm{C}(E)$, both $p^{+}$and $p^{-}$are convex, thus there exist elements $u(p) \in p^{+}$ and $v(p) \in p^{-}$such that

$$
((x(p)-r(p))+(y(p)-s(p))) u(p)=(x(p)-r(p)) u^{\prime}(p)+(y(p)-s(p)) u^{\prime \prime}(p)
$$

and

$$
((y(p)-r(p))+(z(p)-s(p))) v(p)=(y(p)-r(p)) v^{\prime}(p)+(z(p)-s(p)) v^{\prime \prime}(p),
$$

whence, adding together (1.1) and (1.2), we obtain

$$
\sum_{p}(x(p)+y(p)-r(p)-s(p)) u(p) \leq \sum_{p}(y(p)+z(p)-r(p)-s(p)) v(p)
$$

which can be written

$$
\sum_{p}(x(p)-t(p)) u(p) \leq \sum_{p}(z(p)-t(p)) v(p)
$$

where $t=r+s-y$. Since $t \leq x, z$, we obtain $x \sqsubseteq z$.

Therefore, by Lemmas 1.5 to 1.7 , the relation $\sqsubseteq$ is a $K$-vector space preordering. Since it is not in general antisymmetric, we shall consider the associated equivalence relation, that is, the binary relation $\equiv$ defined by $x \equiv y \Leftrightarrow(x \sqsubseteq y$ and $y \sqsubseteq x)$.

Definition 1.8. Let $\mathbf{I}_{K}(E)$ be the quotient ordered vector space $\left(K^{(\mathrm{C}(E))},+, 0, \sqsubseteq\right) / \equiv$. We will denote by $\pi_{E}$ the natural projection from $K^{(\mathrm{C}(E))}$ onto $\mathbf{I}_{K}(E)$.

We will now define an embedding from $E$ into $\mathbf{I}_{K}(E)$. For all $x \in E$, let $\dot{x}=\langle x, x, x, x\rangle \in$ $\mathrm{C}(E)$, identified as usual with the corresponding element of $K^{(\mathrm{C}(E))}$; then put $i_{E}(x)=$ $\pi_{E}(\dot{x})$.

Lemma 1.9. The mapping $i_{E}$ is a cofinal embedding of ordered $K$-vector spaces from $E$ into $\mathbf{I}_{K}(E)$.

Proof. We begin by proving that $i_{E}$ is a $K$-linear map. The proof proceeds in four steps.

(a) $\dot{0} \equiv 0$ (the first 0 is the one of $E$, the second 0 is the one of $\left.K^{(\mathrm{C}(E))}\right)$. It suffices to verify that $0 \ll \dot{0}$ and $\dot{0} \ll 0$; this is immediate.

(b) For all $x \in E$ and all $\lambda \in K^{+}$, if $y=\lambda x$, then $\dot{y} \equiv \lambda \dot{x}$. Indeed, since both $\dot{y}$ and $\lambda \dot{x}$ belong to $\left(K^{+}\right)^{(\mathrm{C}(E))}$, it suffices to prove that $\dot{y} \ll \lambda \dot{x}$ and $\lambda \dot{x} \ll \dot{y}$. Since $x \in \dot{x}^{+} \cap \dot{x}^{-}$ and $\lambda x=y \in \dot{y}^{+} \cap \dot{y}^{-}$, this is immediate. 
(c) For all $x$ and $y$ in $E$, if $z=x+y$, then $\dot{z} \equiv \dot{x}+\dot{y}$. Indeed, since $\dot{x}, \dot{y}$ and $\dot{z}$ belong to $\left(K^{+}\right)^{(\mathrm{C}(E))}$, it suffices to verify that $\dot{z} \ll \dot{x}+\dot{y}$ and $\dot{x}+\dot{y} \ll \dot{z}$. Again, this is immediate.

(d) For all $x \in E$ and all $\lambda \in K$, if $y=\lambda x$, then $\dot{y} \equiv \lambda \dot{x}$. Indeed, we have already seen in (b) that this is true for $\lambda \geq 0$, so suppose now that $\lambda<0$ and put $\mu=-\lambda$. Put $z=-y$. Since $y+z=0$ and by (a) and (c), we have $\dot{y}+\dot{z} \equiv \dot{0} \equiv 0$. Moreover, by (b), $\dot{z} \equiv \mu \dot{x}$, whence $\dot{y} \equiv-\dot{z} \equiv-(\mu \dot{x})=\lambda \dot{x}$.

At this point, we have verified that $i_{E}$ is $K$-linear. Further, let $x \in E$; if $0 \leq x$, then trivially $0 \ll \dot{x}$ thus $0 \sqsubseteq \dot{x}$; conversely, if $0 \sqsubseteq \dot{x}$, then, by Lemma $1.4,0 \ll \dot{x}$, thus $0 \leq x$ by definition, and so we have proved that $i_{E}$ is an order-embedding.

Finally, every element of $\mathbf{I}_{K}(E)$ is a $K$-linear combination of classes modulo $\equiv$ of elements of $\mathrm{C}(E)$, thus, to prove that $i_{E}$ is cofinal, it suffices to prove that for all $p \in \mathrm{C}(E)$ and all $\lambda \in K$, there exists $c \in E$ such that $\lambda p \sqsubseteq \dot{c}$; if $\lambda \geq 0$ it suffices to take $c=\lambda p(2)$, and if $\lambda<0$ it suffices to take $c=\lambda p(0)$.

Now that we know that $i_{E}$ is an embedding of ordered vector spaces, we shall as usual identify $E$ with its image in $\mathbf{I}_{K}(E)$ under $i_{E}$. Now let us put, for all $p \in \mathrm{C}(E), \bowtie(p)=$ $\pi_{E}(p)$; note that if $p$ is trivial, then $\bowtie(p) \in E$ : indeed, if for example $p(1)=p(2)=c$, then $\dot{c} \equiv p$, thus $\bowtie(p)=c$. Therefore, every element of $\mathbf{I}_{K}(E)$ can be written as a finite sum $a+\sum_{i<n} \lambda_{i} \cdot \bowtie\left(p_{i}\right)$ where $a \in E, n \in \omega$, all $p_{i}(i<n)$ are non trivial elements of $\mathrm{C}(E)$ and all $\lambda_{i}(i<n)$ belong to $K$. It is not difficult to verify that this expression is in fact unique, but this will not be needed.

Unfortunately, $\mathbf{I}_{K}(E)$ may not satisfy interpolation; nevertherless, it is the first step of the construction of an extension of $E$ satisfying interpolation:

Lemma 1.10. The space $\mathbf{I}_{K}(E)$ interpolates $E$, i.e., for all $p \in \mathrm{C}(E), p$ admits an interpolant in $\mathbf{I}_{K}(E)$.

Proof. It is easy to verify that $\bowtie(p)$ is an interpolant of $p$ in $\mathbf{I}_{K}(E)$.

Now, we shall see that $\mathbf{I}_{K}$ is in fact a functor - in fact, the correspondence $E \mapsto i_{E}$ is functorial. We need to define $\mathbf{I}_{K}(f)$ for any element $f$ of $\operatorname{Hom}_{K}^{+}(E, F)$ where $E$ and $F$ are arbitrary ordered $K$-vector spaces. Thus let first $\tilde{f}$ be the unique $K$-linear map from $K^{(\mathrm{C}(E))}$ to $K^{(\mathrm{C}(F))}$ such that

$$
(\forall p \in \mathrm{C}(E))(\tilde{f}(p)=f \circ p)
$$

Note that $\tilde{f}$ may send non trivial elements of $\mathrm{C}(E)$ to trivial elements of $\mathrm{C}(F)$ : this is the main reason for considering all, trivial or not, elements of $\mathrm{C}(E)$ within the definition of $\mathbf{I}_{K}(E)$. 
Lemma 1.11. The map $\tilde{f}$ preserves $\leq, \ll$ and $\sqsubseteq$.

Proof. The statement about $\leq$ is trivial. Now, let $x$ and $y$ be elements of $\left(K^{+}\right)^{(\mathrm{C}(E))}$ such that $x \ll y$. By definition, there are $u \in \Sigma(E)$ and $v \in \Pi(E)$ such that $\sum_{p} x(p) u(p) \leq$ $\sum_{p} y(p) v(p)$. Therefore, applying $f$, we obtain $\sum_{p} x(p) f(u(p)) \leq \sum_{p} y(p) f(v(p))$; note that for all $p, f(u(p)) \in(f \circ p)^{+}$and $f(v(p)) \in(f \circ p)^{-}$. By Lemma 1.2, this implies immediately that $\sum_{p} x(p)(f \circ p) \ll \sum_{p} y(p)(f \circ p)$, i.e., $\tilde{f}(x) \ll \tilde{f}(y)$; thus the statement about $\ll$.

Finally, if $x$ and $y$ are elements of $K^{(\mathrm{C}(E))}$ such that $x \sqsubseteq y$, then, by definition, there exists $z \leq x, y$ in $K^{(\mathrm{C}(E))}$ such that $x-z \ll y-z$. By the previous paragraph, $\tilde{f}(x-z) \ll$ $\tilde{f}(y-z)$, i.e., $\tilde{f}(x)-\tilde{f}(z) \ll \tilde{f}(y)-\tilde{f}(z)$. Since we have seen that $\tilde{f}$ is $\leq$-preserving, we have $\tilde{f}(z) \leq \tilde{f}(x), \tilde{f}(y)$, whence $\tilde{f}(x) \sqsubseteq \tilde{f}(y)$.

Therefore, there exists a unique positive homomorphism $\mathbf{I}_{K}(f): \mathbf{I}_{K}(E) \rightarrow \mathbf{I}_{K}(F)$ making the following diagram commute:

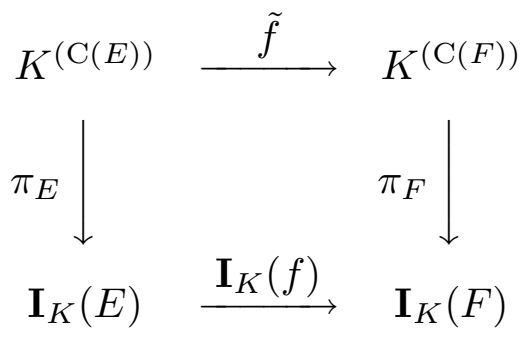

The effect of $\mathbf{I}_{K}(f)$ on the elements of $\mathbf{I}_{K}(E)$ may be described by the formula

$$
\mathbf{I}_{K}(f)\left(a+\sum_{p} \lambda_{p} \cdot \bowtie(p)\right)=f(a)+\sum_{p} \lambda_{p} \cdot \bowtie(f \circ p) .
$$

The following easy lemma shows that $\mathbf{I}_{K}(f)$ defines not only a morphism from $\mathbf{I}_{K}(E)$ to $\mathbf{I}_{K}(F)$, but in fact a morphism from $i_{E}$ to $i_{F}$ :

Lemma 1.12. The following diagram commutes:

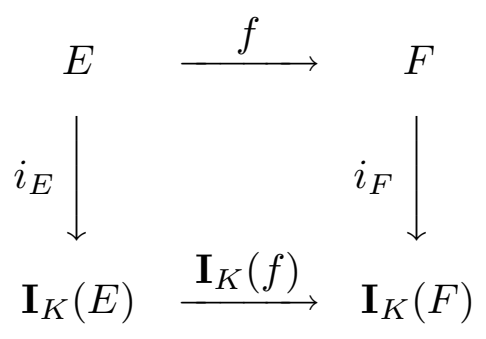


The following easy lemma shows in particular that $\mathbf{I}_{K}$ is indeed a functor:

Lemma 1.13. Let $f: E \rightarrow F$ and $g: F \rightarrow G$ be positive homomorphisms. Then $\widetilde{g \circ f}=$ $\tilde{g} \circ \tilde{f}$ and $\mathbf{I}_{K}(g \circ f)=\mathbf{I}_{K}(g) \circ \mathbf{I}_{K}(f)$.

It can also be proved that the functor $\mathbf{I}_{K}$ preserves the notion of embedding; however, all the embeddings which we shall use will in fact have retractions, thus we will not need this result in this paper.

Lemma 1.14. The functor $\mathbf{I}_{K}$ preserves direct limits.

Proof. A tedious but essentially routine proof. Suppose that an ordered $K$-vector space $E$ is a direct limit of a direct system $\left(E_{i}, f_{i j}\right)_{i \leq j}$ in $I$ (where $I$ is a directed partially ordered set) with limiting maps $f_{i}: E_{i} \rightarrow E$. Note that such direct limits are characterized by $E=\bigcup_{i \in I} f_{i}\left[E_{i}\right]$ and for all $i \in I$ and $x \in E_{i}, f_{i}(x) \geq 0 \Leftrightarrow(\exists j \geq i)\left(f_{i j}(x) \geq 0\right)$ (compare with [11, Proposition 1.15]). We check that these conditions are satisfied by the image of the direct system under the functor $\mathbf{I}_{K}$.

Thus let first $x$ be an element of $K^{(\mathrm{C}(E))}$. One can write $x=\sum_{j<n} \lambda_{j} p_{j}$ for some $n \in \omega$, $\lambda_{j} \in K$ and $p_{j} \in \mathrm{C}(E)$ (all $\left.j<n\right)$. Since $E$ is the direct limit of the $E_{i}$ 's, there exists $i \in I$ such that all the $p_{j}$ 's are of the form $f_{i} \circ q_{j}$ for some $q_{j} \in \mathrm{C}\left(E_{i}\right)$; whence $x=\tilde{f}_{i}(y)$ where $y=\sum_{j<n} \lambda_{j} q_{j}$. Thus $K^{(\mathrm{C}(E))}=\bigcup_{i \in I} \tilde{f}_{i}\left[K^{\left(\mathrm{C}\left(E_{i}\right)\right)}\right]$. It follows immediately that $\mathbf{I}_{K}(E)=\bigcup_{i \in I} \mathbf{I}_{K}\left(f_{i}\right)\left[\mathbf{I}_{K}\left(E_{i}\right)\right]$.

Next, we prove that for every $i \in I$ and every element $x$ of $K^{\left(\mathrm{C}\left(E_{i}\right) \text { ) }\right.}, \tilde{f}_{i}(x) \geq 0$ (for the componentwise ordering) implies that there exists $j \geq i$ such that $\tilde{f}_{i j}(x) \geq 0$. Indeed, write $x=\sum_{p \in S} x_{p} p$ where $S$ is a finite subset of $\mathrm{C}\left(E_{i}\right)$. Thus $\tilde{f}_{i}(x) \geq 0$ means that for every $q \in S, \sum_{p \in S \mid f_{i} \circ p=f_{i} \circ q} x_{p} \geq 0$. But since $S$ is finite, there exists $j \geq i$ such that for all elements $p$ and $q$ of $S, f_{i} \circ p=f_{i} \circ q$ if and only if $f_{i j} \circ p=f_{i j} \circ q$; thus $\tilde{f}_{i j}(x) \geq 0$.

Now let us prove that for any $i \in I$ and all elements $x$ and $y$ of $\left(K^{+}\right)^{\left(\mathrm{C}\left(E_{i}\right)\right)}, \tilde{f}_{i}(x) \ll \tilde{f}_{i}(y)$ implies that there exists $j \geq i$ in $I$ such that $\tilde{f}_{i j}(x) \ll \tilde{f}_{i j}(y)$. Indeed, by Lemma 1.2 , there exist $\bar{u} \in \prod_{p \in \mathrm{C}\left(E_{i}\right)}\left(f_{i} \circ p\right)^{+}$and $\bar{v} \in \prod_{p \in \mathrm{C}\left(E_{i}\right)}\left(f_{i} \circ p\right)^{-}$such that $\sum_{p \in \mathrm{C}\left(E_{i}\right)} x(p) \bar{u}(p) \leq$ $\sum_{p \in \mathrm{C}\left(E_{i}\right)} y(p) \bar{v}(p)$. Now for all $p$, one can write $\bar{u}(p)=f_{i}(u(p))$ and $\bar{v}(p)=f_{i}(v(p))$ for some $u(p) \in p^{+}$and $v(p) \in p^{-}$. Therefore, one obtains $f_{i}\left(\sum_{p \in \mathrm{C}\left(E_{i}\right)} x(p) u(p)\right) \leq$ $f_{i}\left(\sum_{p \in \mathrm{C}\left(E_{i}\right)} y(p) v(p)\right)$, thus, since $E$ is the direct limit of the $E_{j}$ 's, there exists $j \geq i$ in $I$ such that $f_{i j}\left(\sum_{p} x(p) u(p)\right) \leq f_{i j}\left(\sum_{p} y(p) v(p)\right)$, i.e., $\sum_{p} x(p) f_{i j}(u(p)) \leq \sum_{p} y(p) f_{i j}(v(p))$; therefore, by Lemma $1.2, \tilde{f}_{i j}(x) \ll \tilde{f}_{i j}(y)$.

Finally, to conclude, it suffices to prove that for any $i \in I$ and all elements $x$ and $y$ of $K^{\left(\mathrm{C}\left(E_{i}\right)\right)}, \tilde{f}_{i}(x) \sqsubseteq \tilde{f}_{i}(y)$ implies that there exists $j \geq i$ in $I$ such that $\tilde{f}_{i j}(x) \sqsubseteq$ 
$\tilde{f}_{i j}(y)$ (it does not bring any simplification to restrict to the case $x=0$ ). By definition, there exists $z \in K^{(\mathrm{C}(E))}$ such that $z \leq \tilde{f}_{i}(x), \tilde{f}_{i}(y)$ and $\tilde{f}_{i}(x)-z \ll \tilde{f}_{i}(y)-z$. By the result of the second paragraph, one can write $z=\tilde{f}_{j}\left(z^{\prime}\right)$ for some $j \geq i$ and some $z^{\prime} \in E_{j}$. By the result of the third paragraph, one can suppose (after having taken if needed a larger value for $j$ ) that $z^{\prime} \leq \tilde{f}_{i j}(x), \tilde{f}_{i j}(y)$. Thus, using Lemma 1.13, we obtain $\tilde{f}_{j}\left(\tilde{f}_{i j}(x)-z^{\prime}\right) \ll \tilde{f}_{j}\left(\tilde{f}_{i j}(y)-z^{\prime}\right)$, whence, by the previous paragraph, there exists $k \geq j$ such that $\tilde{f}_{j k}\left(\tilde{f}_{i j}(x)-z^{\prime}\right) \ll \tilde{f}_{j k}\left(\tilde{f}_{i j}(y)-z^{\prime}\right)$, which can be written $\tilde{f}_{i k}(x)-\tilde{f}_{j k}\left(z^{\prime}\right) \ll$ $\tilde{f}_{i k}(y)-\tilde{f}_{j k}\left(z^{\prime}\right)$. Since $\tilde{f}_{j k}$ preserves $\leq$, we have $\tilde{f}_{j k}\left(z^{\prime}\right) \leq \tilde{f}_{i k}(x), \tilde{f}_{i k}(y)$ and thus, by definition, $\tilde{f}_{i k}(x) \sqsubseteq \tilde{f}_{i k}(y)$. This implies immediately that for all $i \in I$ and all $x \in \mathbf{I}_{K}\left(E_{i}\right)$, $\mathbf{I}_{K}\left(f_{i}\right)(x) \geq 0$ implies that there exists $j \geq i$ such that $\mathbf{I}_{K}\left(f_{i j}\right)(x) \geq 0$.

We shall now define, for every ordered $K$-vector space $E$, an interpolation vector space $\mathbf{J}_{K}(E)$ containing $E$ : simply put $\mathbf{I}_{K}^{0}(E)=E, \mathbf{I}_{K}^{n+1}(E)=\mathbf{I}_{K}\left(\mathbf{I}_{K}^{n}(E)\right)$ for all $n \in \omega$, and $\mathbf{J}_{K}(E)=\bigcup_{n \in \omega} \mathbf{I}_{K}^{n}(E)$. Denote by $j_{E}$ the inclusion map from $E$ into $\mathbf{J}_{K}(E)$. As an immediate consequence of Lemmas 1.9 and 1.10, we have

Proposition 1.15. The space $\mathbf{J}_{K}(E)$ is an interpolation vector space, in which $E$ is cofinal.

The way in which $\mathbf{J}_{K}$ is made a functor is just as natural: if $f: E \rightarrow F$ is a positive homomorphism, put $\mathbf{I}_{K}^{0}(f)=f, \mathbf{I}_{K}^{n+1}(f)=\mathbf{I}_{K}\left(\mathbf{I}_{K}^{n}(f)\right)$ for all $n \in \omega$, and $\mathbf{J}_{K}(f)=$ $\bigcup_{n \in \omega} \mathbf{I}_{K}^{n}(f)$. Then it is pure routine to verify both of the following results:

Proposition 1.16. For every positive homomorphism $f: E \rightarrow F$, the following diagram commutes:

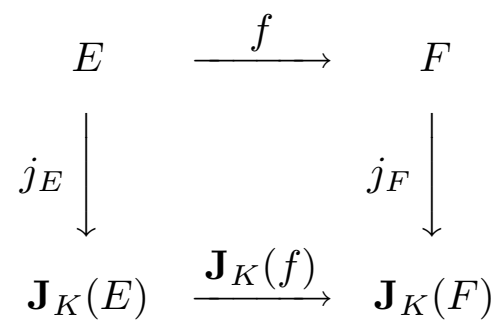

Proof. Use Lemma 1.12.

Proposition 1.17. $\mathbf{J}_{K}$ is a direct limits preserving functor.

Proof. Use Lemmas 1.13 to 1.14, and commutation of direct limits.

The following notion will allow us to get some control over the elements of $\mathbf{J}_{K}(E)$. 
Definition 1.18. Let $E$ be an ordered $K$-vector space. An interpolator on $E$ is a map $\iota: \mathrm{C}(E) \rightarrow E$ such that

$$
(\forall p \in \mathrm{C}(E))(p(0), p(1) \leq \iota(p) \leq p(2), p(3)) .
$$

Note that $E$ admits an interpolator if and only if $E$ is an interpolation vector space. In the next section, the main part of the argument will be to choose a very special interpolator on a certain interpolation vector space.

The following lemma will allow us to extend morphisms coherently with respect to a given interpolator.

Lemma 1.19. Let $E$ and $G$ be ordered $K$-vector spaces and let $\iota$ be an interpolator on $G$. Then every element $f$ of $\operatorname{Hom}_{K}^{+}(E, G)$ extends to a unique element $f_{(\iota)}$ of $\operatorname{Hom}_{K}^{+}\left(\mathbf{I}_{K}(E), G\right)$ such that

$$
(\forall p \in \mathrm{C}(E))\left(f_{(\iota)}(\bowtie(p))=\iota(f \circ p)\right) .
$$

Furthermore, if $H$ is a subspace of $G$ closed under $\iota$ (i.e., $\iota[\mathrm{C}(H)] \subseteq H$ ) such that $\operatorname{rng}(f) \subseteq$ $H$, then $\operatorname{rng}\left(f_{(\iota)}\right) \subseteq H$.

Proof. Since $\mathbf{I}_{K}(E)$ is generated by the $\bowtie(p)$ 's as a vector space, uniqueness is trivial. Now let $g: K^{(\mathrm{C}(E))} \rightarrow G$ be the $K$-linear map defined by $g(p)=\iota(f \circ p)($ all $p \in \mathrm{C}(E))$.

Let first $x$ and $y$ be elements of $\left(K^{+}\right)^{(\mathrm{C}(E))}$, we prove that $x \ll y$ implies that $g(x) \leq$ $g(y)$. Indeed, by definition, there exist $u \in \Sigma(E)$ and $v \in \Pi(E)$ such that $\sum_{p} x(p) u(p) \leq$ $\sum_{p} y(p) v(p)$. Applying $f$ yields $\sum_{p} x(p) f(u(p)) \leq \sum_{p} y(p) f(v(p))$. But for all $p \in \mathrm{C}(E)$, $f(v(p)) \in(f \circ p)^{-}$and $f(u(p)) \in(f \circ p)^{+}$, whence $f(v(p)) \leq \iota(f \circ p) \leq f(u(p))$; therefore, since the $x(p)$ 's and the $y(p)$ 's belong to $K^{+}$, we obtain $\sum_{p} x(p) \iota(f \circ p) \leq \sum_{p} y(p) \iota(f \circ p)$, i.e., $g(x) \leq g(y)$.

Now, if $x$ and $y$ are two elements of $K^{(\mathrm{C}(E))}$ such that $x \sqsubseteq y$, there exists by definition $z \leq x, y$ in $K^{(\mathrm{C}(E))}$ such that $x-z \ll y-z$, whence, by the previous paragraph, $g(x-z) \leq$ $g(y-z)$, thus, since $g$ is linear, $g(x) \leq g(y)$.

Hence, there exists a [unique] element $f_{(\iota)}$ of $\operatorname{Hom}_{K}^{+}\left(\mathbf{I}_{K}(E), G\right)$ such that $f_{(\iota)} \circ \pi_{E}=g$ (recall that $\pi_{E}$ is the natural projection from $K^{(\mathrm{C}(E))}$ onto $\mathbf{I}_{K}(E)$ ). In particular, for all $p \in \mathrm{C}(E), f_{(\iota)}(\bowtie(p))=f_{(\iota)} \circ \pi_{E}(p)=g(p)=\iota(f \circ p)$. The last part of the statement of the lemma is trivial.

The aforementioned coherence can now fully express itself in the following lemma:

Lemma 1.20. Let $E, F$ and $G$ be ordered $K$-vector spaces, let $\iota$ be an interpolator on $G$. Let $f \in \operatorname{Hom}_{K}^{+}(F, G)$ and $\varphi \in \operatorname{Hom}_{K}^{+}(E, F)$. Then $(f \circ \varphi)_{(\iota)}=f_{(\iota)} \circ \mathbf{I}_{K}(\varphi)$. 
Proof. It is sufficient to check the equality on those elements of the form $\bowtie(p)$ where $p \in \mathrm{C}(E)$; we proceed: $f_{(\iota)} \circ \mathbf{I}_{K}(\varphi)(\bowtie(p))=f_{(\iota)}(\bowtie(\varphi \circ p))=\iota(f \circ \varphi \circ p)=(f \circ \varphi)_{(\iota)}(\bowtie(p))$.

Now, the extension process described in Lemma 1.19 can be repeated to yield an extension from $\mathbf{J}_{K}(E)$ to $G$ : formally, if $\iota$ is an interpolator on $G$ and $f \in \operatorname{Hom}_{K}^{+}(E, G)$, define $f_{0}=f$ and $f_{n+1}=\left(f_{n}\right)_{(\iota)}$ for all $n \in \omega$, so that for all $n, f_{n} \in \operatorname{Hom}_{K}^{+}\left(\mathbf{I}_{K}^{n}(E), G\right)$. Then, put $f_{[\iota]}=\bigcup_{n \in \omega} f_{n}$. Then a routine checking yields us the following extensions of Lemmas 1.19 and 1.20 :

Proposition 1.21. Let $E$ and $G$ be ordered $K$-vector spaces and let $\iota$ be an interpolator on $G$. Then for every $f \in \operatorname{Hom}_{K}^{+}(E, G), f_{[\ell]}$ is an extension of $f$ which belongs to $\operatorname{Hom}_{K}^{+}\left(\mathbf{J}_{K}(E), G\right)$. Furthermore, if $H$ is a subspace of $G$ closed under $\iota$ such that $\operatorname{rng}(f) \subseteq H$, then $\operatorname{rng}\left(f_{[\iota]}\right) \subseteq H$.

This shows in particular that $\mathbf{J}_{K}(E)$ is a "quasi-universal" interpolation vector space above $E$, in the sense that every positive homomorphism from $E$ to an interpolation vector space $G$ extends to a positive homomorphism from $\mathbf{J}_{K}(E)$ to $G$, although not necessarily in a unique way. Nevertheless, the choice of an interpolator allows us to find a canonical extension of the positive homomorphism under question.

Proposition 1.22. Let $E, F$ and $G$ be ordered $K$-vector spaces, let $\iota$ be an interpolator on $G$. Let $f \in \operatorname{Hom}_{K}^{+}(F, G)$ and $\varphi \in \operatorname{Hom}_{K}^{+}(E, F)$. Then $(f \circ \varphi)_{[\iota]}=f_{[\iota]} \circ \mathbf{J}_{K}(\varphi)$.

\section{$\S 2$. Construction of the functors $\mathbf{E}_{K}$ and $\mathbf{F}_{K}$.}

From 2.1 to $2.7, K$ will again be a given (totally) ordered field.

Definition 2.1. For every set $X$, let $\mathbf{E}_{K}(X)$ be the partially ordered $K$-vector space defined by generators $a_{x}^{X}($ all $x \in X)$ and $e^{X}$ and relations $0 \leq a_{x}^{X} \leq e^{X}$ (all $\left.x \in X\right)$.

In the context of the Definition above, we will put $b_{x}^{X}=e^{X}-a_{x}^{X}$. Note that for all $x_{0} \in X$, there exists a unique positive homomorphism $f_{x_{0}}: \mathbf{E}_{K}(X) \rightarrow K$ such that $f_{x_{0}}\left(e^{X}\right)=f_{x_{0}}\left(a_{x_{0}}^{X}\right)=1$ and $f_{x_{0}}\left(a_{x}^{X}\right)=0$ for all $x \in X \backslash\left\{x_{0}\right\}$; similarly, there exists a unique positive homomorphism $f: \mathbf{E}_{K}(X) \rightarrow K$ such that $f\left(e^{X}\right)=1$ and $f\left(a_{x}^{X}\right)=0$ for all $x \in X$. Therefore, one deduces easily the fact that the family consisting of $e^{X}$ together with all $a_{x}^{X}(x \in X)$ is a basis of $\mathbf{E}_{K}(X)$ as a $K$-vector space. The proof of the following lemma is easy routine: 
Lemma 2.2. Let $X$ and $Y$ be two sets and let $f: X \rightarrow Y$ be any mapping. Then there exists a unique positive homomorphism $\mathbf{E}_{K}(f): \mathbf{E}_{K}(X) \rightarrow \mathbf{E}_{K}(Y)$ such that

$$
\mathbf{E}_{K}(f)\left(e^{X}\right)=e^{Y} \quad \text { and } \quad(\forall x \in X)\left(\mathbf{E}_{K}(f)\left(a_{x}^{X}\right)=a_{f(x)}^{Y}\right) .
$$

Moreover, $\mathbf{E}_{K}$ thus defined is a direct limits preserving functor from the category of sets to the category of ordered $K$-vector spaces.

In particular, if $Y$ is a subset of a set $X$, denote by $e_{Y X}$ the image under $\mathbf{E}_{K}$ of the inclusion map from $Y$ into $X$. One can also, in the same context, define a positive homomorphism from $\mathbf{E}_{K}(X)$ to $\mathbf{E}_{K}(Y)$ :

Lemma 2.3. Let $X$ be a set, let $Y$ be a subset of $X$. Then there exists a unique positive homomorphism $r_{Y}^{X}: \mathbf{E}_{K}(X) \rightarrow \mathbf{E}_{K}(Y)$ such that

$$
r_{Y}^{X}\left(e^{X}\right)=e^{Y} \quad \text { and } \quad(\forall x \in Y)\left(r_{Y}^{X}\left(a_{x}^{X}\right)=a_{x}^{Y}\right) \quad \text { and } \quad(\forall x \in X \backslash Y)\left(r_{Y}^{X}\left(a_{x}^{X}\right)=0\right) .
$$

Note that trivially, $r_{Y}^{X} \circ e_{Y X}=\operatorname{id}_{\mathbf{E}_{K}(Y)}$. Therefore, $e_{Y X}$ is an embedding of ordered $K$-vector spaces from $\mathbf{E}_{K}(Y)$ into $\mathbf{E}_{K}(X)$ - it has even $r_{Y}^{X}$ as retraction (even when $Y$ is empty; note that $\left.\mathbf{E}_{K}(\emptyset) \cong K\right)$. Thus from now on, we shall drop the superscript $X$ from $a_{x}^{X}, b_{x}^{X}$ and $e^{X}$, keeping the notations $a_{x}, b_{x}, e$. In particular, for $Y \subseteq X$, we shall identify $\mathbf{E}_{K}(Y)$ with its natural image in $\mathbf{E}_{K}(X)$.

Definition 2.4. Let $\mathbf{F}_{K}=\mathbf{J}_{K} \mathbf{E}_{K}$ be the composition of both functors $\mathbf{J}_{K}$ (of Section 1) and $\mathbf{E}_{K}$.

If $Y$ is a subset of $X$, we shall put $f_{Y X}=\mathbf{J}_{K}\left(e_{Y X}\right)$ and $s_{Y}^{X}=\mathbf{J}_{K}\left(r_{Y}^{X}\right)$. Since $s_{Y}^{X} \circ f_{Y X}=$ $\operatorname{id}_{\mathbf{F}_{K}(Y)}, f_{Y X}$ is an embedding from $\mathbf{F}_{K}(Y)$ into $\mathbf{F}_{K}(X)$, with $s_{Y}^{X}$ as retraction.

By Proposition 1.17 and Lemma 2.2, the functor $\mathbf{F}_{K}$ preserves direct limits. This shows in particular that for any set $X, \mathbf{F}_{K}(X)$ is the direct limit of all $\mathbf{F}_{K}(S)$ for $S$ ranging over finite subsets of $X$, with the obvious transition maps and limiting maps. Thus every element $x$ of $\mathbf{F}_{K}(X)$ belongs to some $f_{S X}\left[\mathbf{F}_{K}(S)\right]$ for some finite subset $S$ of $X$. Say that a support of $x$ is any subset $S$ of $X$ such that $x \in f_{S X}\left[\mathbf{F}_{K}(S)\right]$ : therefore, every element of $\mathbf{F}_{K}(X)$ admits a [and in general more than one] finite support. Note that the set of all supports of $X$ is upward directed for the inclusion (among all subsets of $X$ ).

We shall now state a set-theoretical result which is in fact an easy translation of one of the directions of the result of $[\mathbf{1 8}]$ (and in fact, only the particular case $n=2$ will be needed); since the proof is easy, we include one for convenience: 
Proposition 2.5. Let $n$ be a non negative integer, let $S$ be a set of cardinality at least $\aleph_{n}$ and let $f:[S]^{n} \rightarrow[S]^{<\omega}$ be any mapping. Then there exists $U \in[S]^{n+1}$ such that $(\forall x \in U)(x \notin f(U \backslash\{x\}))$.

Proof. By induction on $n$. If $n=0$ it is trivial, thus suppose that $n>0$. Without loss of generality, $\omega_{n} \subseteq S$, and, by considering $f^{\prime}:\left[\omega_{n}\right]^{n} \rightarrow\left[\omega_{n}\right]^{<\omega}$ defined by $f^{\prime}(X)=f(X) \cap \omega_{n}$, one can suppose without loss of generality that $S=\omega_{n}$. Since $\omega_{n}$ is a regular cardinal, one can define a strictly increasing function $\Phi: \omega_{n} \rightarrow \omega_{n}$ by putting

$$
\Phi(\xi)=\text { least } \kappa<\omega_{n} \text { such that } \Phi[\xi] \subseteq \kappa \text { and }\left(\forall X \in[\xi]^{n}\right)(f(\Phi[X]) \subseteq \kappa)
$$

In particular, $T=\Phi\left[\omega_{n-1}\right]$ is a subset of $S$ of cardinality $\aleph_{n-1}$. Now let $g:[T]^{n-1} \rightarrow[T]^{<\omega}$ be the function defined by

$$
\left(\forall Y \in[T]^{n-1}\right)\left(g(Y)=f\left(Y \cup\left\{\Phi\left(\omega_{n-1}\right)\right\}\right) \cap T\right)
$$

By the induction hypothesis, there exists $V \in[T]^{n}$ such that

$$
(\forall y \in V)(y \notin g(V \backslash\{y\}))
$$

Put $U=V \cup\left\{\Phi\left(\omega_{n-1}\right)\right\}$. Thus $U \in[S]^{n+1}$, and it is easy to verify that $U$ satisfies the desired conclusion.

Note that the other direction of $[\mathbf{1 8}]$ shows in fact that the bound $\aleph_{n}$ is optimal for the result (which probably justifies the title of $[\mathbf{1 8}]$ ).

In order to state our main lemma, denote, for all $i<3$, by $i^{\prime}$ and $i^{\prime \prime}$ both elements of $3 \backslash\{i\}$ arranged in such a way that $i^{\prime}<i^{\prime \prime}$. Next, for all $i<3$, define

$$
c_{i}=\bowtie\left(p_{i}\right) \quad \text { where } \quad p_{i}=\left\langle 0, a_{i^{\prime}}+a_{i^{\prime \prime}}-e, a_{i^{\prime}}, a_{i^{\prime \prime}}\right\rangle
$$

(whether we put $i^{\prime}$ first and then $i^{\prime \prime}$ is arbitrary, the converse would also work). For all $i<3$, let $G_{i}$ be the subspace of $\mathbf{F}_{K}(3)$ generated by $\left\{a_{i^{\prime}}, b_{i^{\prime}}, a_{i^{\prime \prime}}, b_{i^{\prime \prime}}, c_{i}\right\}$, let $\varphi_{i}$ be the map from $K^{4}$ to $G_{i}$ defined by the formula

$$
\varphi_{i}\left(\left\langle x_{0}, x_{1}, x_{2}, x_{3}\right\rangle\right)=x_{0} c_{i}+x_{1}\left(a_{i^{\prime}}-c_{i}\right)+x_{2}\left(a_{i^{\prime \prime}}-c_{i}\right)+x_{3}\left(c_{i}+e-a_{i^{\prime}}-a_{i^{\prime \prime}}\right)
$$

and let $\psi_{i}$ be the map from $K^{2}$ to $G_{i^{\prime}} \cap G_{i^{\prime \prime}}$ defined by the formula

$$
\psi_{i}(\langle x, y\rangle)=x a_{i}+y b_{i}
$$


We are indebted to K. Goodearl for a substantial simplification in the following proof:

Lemma 2.6. The following holds:

(i) For all $i<3, \varphi_{i}$ is an isomorphism (of ordered $K$-vector spaces) from $K^{4}$ onto $G_{i}$; thus, $G_{i}$ is lattice-ordered.

(ii) For all $i<3, \psi_{i}$ is an isomorphism (of ordered $K$-vector spaces) from $K^{2}$ onto $G_{i^{\prime}} \cap G_{i^{\prime \prime}}$.

(iii) $G_{0} \cap G_{1} \cap G_{2}=K e$.

Proof. (i) We prove it for example for $i=0$, the proofs for $i=1$ and $i=2$ being similar. First, since $c_{0}$ is an interpolant of $p_{0}=\left\langle 0, a_{1}+a_{2}-e, a_{1}, a_{2}\right\rangle$ (Lemma 1.10), it is obvious that $\varphi_{0}$ is a positive homomorphism. Next, $\varphi_{0}$ is surjective: indeed, it is immediate to verify that

$$
\begin{aligned}
& a_{1}=\varphi_{0}(\langle 1,1,0,0\rangle), \\
& a_{2}=\varphi_{0}(\langle 1,0,1,0\rangle), \\
& b_{1}=\varphi_{0}(\langle 0,0,1,1\rangle), \\
& b_{2}=\varphi_{0}(\langle 0,1,0,1\rangle), \\
& c_{0}=\varphi_{0}(\langle 1,0,0,0\rangle) .
\end{aligned}
$$

So let us now prove that $\varphi_{0}$ is an embedding. We have seen in 2.1 that $\left\langle a_{0}, a_{1}, a_{2}, e\right\rangle$ is a basis of $\mathbf{E}_{K}(3)$ (as a $K$-vector space), thus there exists a unique $K$-linear map $\sigma_{0}: \mathbf{E}_{K}(3) \rightarrow$ $K^{4}$ sending $a_{0}$ to $\langle 0,0,0,0\rangle, a_{1}$ to $\langle 1,1,0,0\rangle, a_{2}$ to $\langle 1,0,1,0\rangle$ and $e$ to $\langle 1,1,1,1\rangle$. Since $0 \leq \sigma_{0}\left(a_{i}\right) \leq \sigma_{0}(e)$ for all $i<3, \sigma_{0}$ is in fact a positive homomorphism. Moreover, since $K^{4}$ is an interpolation vector space, it admits an interpolator, say $\iota$ (see 1.18). Put $\rho_{0}=\left(\sigma_{0}\right)_{[\iota]}$ (thus $\rho_{0}$ is a positive homomorphism from $\mathbf{F}_{K}(3)$ to $\left.K^{4}\right)$. By definition, $\rho_{0}\left(c_{0}\right)$ interpolates $\sigma_{0} \circ p_{0}=\langle\langle 0,0,0,0\rangle,\langle 1,0,0,-1\rangle,\langle 1,1,0,0\rangle,\langle 1,0,1,0\rangle\rangle$, thus the only possibility is $\rho_{0}\left(c_{0}\right)=\langle 1,0,0,0\rangle$. Therefore, we have

$$
\begin{gathered}
\rho_{0} \circ \varphi_{0}(\langle 1,0,0,0\rangle)=\rho_{0}\left(c_{0}\right)=\langle 1,0,0,0\rangle, \\
\rho_{0} \circ \varphi_{0}(\langle 1,1,0,0\rangle)=\rho_{0}\left(a_{1}\right)=\langle 1,1,0,0\rangle, \\
\rho_{0} \circ \varphi_{0}(\langle 1,0,1,0\rangle)=\rho_{0}\left(a_{2}\right)=\langle 1,0,1,0\rangle, \\
\rho_{0} \circ \varphi_{0}(\langle 1,1,1,1\rangle)=\rho_{0}(e)=\langle 1,1,1,1\rangle,
\end{gathered}
$$

whence $\rho_{0} \circ \varphi_{0}=\mathrm{id}_{K^{4}}$; in particular, $\varphi_{0}$ is an embedding of ordered $K$-vector spaces, as needed to prove part (i).

(ii) Note that for all $x$ and $y$ in $K, \rho_{0} \circ \psi_{1}(\langle x, y\rangle)=\langle x, x, y, y\rangle$, whence $\psi_{1}$ is an embedding of ordered $K$-vector spaces. Now let $z \in G_{0} \cap G_{1}$. By surjectivity of both $\varphi_{0}$ and $\varphi_{1}$ (part 
(i)), there exist elements $x_{i}, y_{i}(i<4)$ of $K$ such that

$$
\begin{aligned}
z & =x_{0} c_{0}+x_{1}\left(a_{1}-c_{0}\right)+x_{2}\left(a_{2}-c_{0}\right)+x_{3}\left(c_{0}+e-a_{1}-a_{2}\right) \\
& =y_{0} c_{1}+y_{1}\left(a_{0}-c_{1}\right)+y_{2}\left(a_{2}-c_{1}\right)+y_{3}\left(c_{1}+e-a_{0}-a_{2}\right) .
\end{aligned}
$$

Now note that $\rho_{0}(0)=\rho_{0}\left(a_{0}\right)=0$ and $0 \leq c_{1} \leq a_{0}$, thus, since $\rho_{0}$ is positive, $\rho_{0}\left(c_{1}\right)=0$, whence, applying $\rho_{0}$ to both displayed equations above, one obtains $\left\langle x_{0}, x_{1}, x_{2}, x_{3}\right\rangle=$ $\left\langle y_{2}, y_{3}, y_{2}, y_{3}\right\rangle$, whence $x_{0}=x_{2}$ and $x_{1}=x_{3}$, thus $z=x_{0} a_{2}+x_{1}\left(e-a_{2}\right)=\psi_{2}\left(\left\langle x_{0}, x_{1}\right\rangle\right)$. Thus $\psi_{2}$ is surjective. Permuting the indexes yields easily part (ii).

For part (iii), let $z \in G_{0} \cap G_{1} \cap G_{2}$. By surjectivity of $\psi_{0}$ and $\psi_{1}$, there are elements $x_{i}, y_{i}(i<2)$ of $K$ such that $z=x_{0} a_{0}+y_{0} b_{0}=x_{1} a_{1}+y_{1} b_{1}$. Thus, applying $\rho_{0}$ yields $\left\langle y_{0}, y_{0}, y_{0}, y_{0}\right\rangle=\left\langle x_{1}, x_{1}, y_{1}, y_{1}\right\rangle$, whence $x_{1}=y_{1}$, so that $z=x_{1} e \in K e$.

Now, we are ready to define an interpolator on $\mathbf{F}_{K}(3)$ : for all $p \in \mathrm{C}\left(\mathbf{F}_{K}(3)\right)$, put $I(p)=\left\{i<3: \operatorname{rng}(p) \subseteq G_{i}\right\}$ and $H_{p}=\bigcap_{i \in I(p)} G_{i}$. Note that $I(p)$ may be empty, in which case we naturally put $H_{p}=\mathbf{F}_{K}(3)$. By Proposition 1.15 and Lemma $2.6, H_{p}$ is an interpolation vector space (it is even lattice-ordered when $I(p) \neq \emptyset$ ), thus $p$ admits an interpolant in $H_{p}$; select any such interpolant $\iota(p)$. Note that by construction, for all $i<3, G_{i}$ is closed under $\iota$. Let $f: \mathbf{E}_{K}(3) \hookrightarrow \mathbf{F}_{K}(3)$ be the inclusion map.

Lemma 2.7. For all $i<3$ and all $x \in \mathbf{F}_{K}(3)$ with support $3 \backslash\{i\}, f_{[\iota]}(x)$ belongs to $G_{i}$.

Proof. By Proposition 1.22 (applied to $G=\mathbf{F}_{K}(3)$ equipped with $\iota, E=\mathbf{E}_{K}(3 \backslash\{i\})$, $F=\mathbf{E}_{K}(3), \varphi=e_{3 \backslash\{i\}, 3}, f$ as defined above), we have $g_{[\iota]}=f_{[\iota]} \circ f_{3 \backslash\{i\}, 3}$ where, by definition, $g=f \circ e_{3 \backslash\{i\}, 3}$. But the range of $g$ is contained in $G_{i}$ and $G_{i}$ is closed under $\iota$, thus, by Proposition 1.21, the range of $g_{[\iota]}$ is also contained into $G_{i}$. Thus if $x \in \mathbf{F}_{K}(3)$ has support $3 \backslash\{i\}$, then, by definition, $x=f_{3 \backslash\{i\}, 3}(y)$ for some $y \in \mathbf{F}_{K}(3 \backslash\{i\})$, whence $f_{[\iota]}(x)=g_{[\iota]}(y) \in G_{i}$.

Now we can prove our main theorem:

Theorem 2.8. Let $K$ be an ordered field, let $\Omega$ be any set of cardinality at least $\aleph_{2}$. Let $\varepsilon$ be an element of $K$ such that $0 \leq \varepsilon<1 / 8$. Then there exist no meet-semilattice $S$ with 1 and no order-preserving map $\mu: S \rightarrow \mathbf{F}_{K}(\Omega)^{+}$satisfying both of the following conditions:

(a) $\mu(1)=e$ and for all $\alpha \in \Omega$, there exists $u_{\alpha} \in S$ such that

$$
a_{\alpha}-\varepsilon e \leq \mu\left(u_{\alpha}\right) \leq a_{\alpha}+\varepsilon e .
$$

(b) For all elements $x, y$ and $z$ of $S$, we have

$$
\mu(x \wedge z)+\mu(y \wedge z) \leq \mu(z)+\mu(x \wedge y)
$$


Proof. In a sequence of claims. For all $\alpha, \beta$ in $\Omega$, put $c_{\alpha \beta}=\mu\left(u_{\alpha} \wedge u_{\beta}\right)$.

Claim 1. For all $\alpha, \beta$ and $\gamma$ in $\Omega$, both following conditions hold:

(i) $c_{\alpha \gamma}+c_{\beta \gamma} \leq a_{\gamma}+\varepsilon e+c_{\alpha \beta}$.

(ii) $0, a_{\alpha}+a_{\beta}-(1+2 \varepsilon) e \leq c_{\alpha \beta} \leq a_{\alpha}+\varepsilon e, a_{\beta}+\varepsilon e$.

Proof of Claim. (i) results from hypothesis (b) with $x=u_{\alpha}, y=u_{\beta}, z=u_{\gamma}$ and then hypothesis (a). Since $\mu$ is order-preserving and positive and by (a), it is trivial that $0 \leq c_{\alpha \beta} \leq a_{\alpha}+\varepsilon e, a_{\beta}+\varepsilon e$. The inequality $a_{\alpha}+a_{\beta} \leq(1+2 \varepsilon) e+c_{\alpha \beta}$ results immediately from hypothesis (b) with $x=u_{\alpha}, y=u_{\beta}$ and $z=1$ and hypothesis (a).

- Claim 1.

For all elements $\alpha$ and $\beta$ of $\Omega$, let $F(\{\alpha, \beta\})$ be a finite support of $c_{\alpha \beta}$ (note that $c_{\alpha \beta}=c_{\beta \alpha}$ so that there is no problem of ordering there). Now, by Proposition 2.5, there exist distinct elements $\alpha, \beta$ and $\gamma$ of $\Omega$ such that $\alpha \notin F(\{\beta, \gamma\}), \beta \notin F(\{\alpha, \gamma\})$ and $\gamma \notin F(\{\alpha, \beta\})$. Throughout the rest of the proof, we shall fix those elements $\alpha, \beta$ and $\gamma$. Put $X=\{\alpha, \beta, \gamma\}$ and let $\tau: X \rightarrow 3$ be the bijection defined by $\tau(\alpha)=0, \tau(\beta)=1$ and $\tau(\gamma)=2$. Then $\mathbf{F}_{K}(\tau)$ is an isomorphism (of ordered $K$-vector spaces) from $\mathbf{F}_{K}(X)$ onto $\mathbf{F}_{K}(3)$. Put $\pi=\mathbf{E}_{K}(\tau) \circ r_{X}^{\Omega}$ and $\phi=\mathbf{J}_{K}(\pi)=\mathbf{F}_{K}(\tau) \circ s_{X}^{\Omega}$ : thus $\pi$ is a positive homomorphism from $\mathbf{E}_{K}(\Omega)$ to $\mathbf{E}_{K}(3)$ and $\phi$ is a positive homomorphism from $\mathbf{F}_{K}(\Omega)$ to $\mathbf{F}_{K}(3)$. Note that $\phi(e)=e, \phi\left(a_{\alpha}\right)=a_{0}, \phi\left(a_{\beta}\right)=a_{1}$ and $\phi\left(a_{\gamma}\right)=a_{2}$. Put $d_{0}=\phi\left(c_{\beta \gamma}\right)$, $d_{1}=\phi\left(c_{\alpha \gamma}\right)$ and $d_{2}=\phi\left(c_{\alpha \beta}\right)$. Note that $d_{0}, d_{1}$ and $d_{2}$ all belong to $\mathbf{F}_{K}(3)^{+}$.

Claim 2. The following holds (in $\left.\mathbf{F}_{K}(3)\right)$ :

(i) $d_{0}+d_{1} \leq a_{2}+\varepsilon e+d_{2}$.

(ii) For all $i<3,0, a_{i^{\prime}}+a_{i^{\prime \prime}}-(1+2 \varepsilon) e \leq d_{i} \leq a_{i^{\prime}}+\varepsilon e, a_{i^{\prime \prime}}+\varepsilon e$.

Proof of Claim. Apply $\phi$ to the inequalities of Claim 1.

Claim 2.

Claim 3. For all $i<3,3 \backslash\{i\}$ is a support of $d_{i}$.

Proof of Claim. We prove it for example for $i=0$. Let $\tau_{0}$ be the restriction of $\tau$ from $\{\beta, \gamma\}$ to $\{1,2\}$ and put $\pi_{0}=\mathbf{E}_{K}\left(\tau_{0}\right) \circ r_{\{\beta, \gamma\}}^{\Omega \backslash\{\alpha\}}, \phi_{0}=\mathbf{J}_{K}\left(\pi_{0}\right)$. Then it is easy to see that the following diagram commutes:

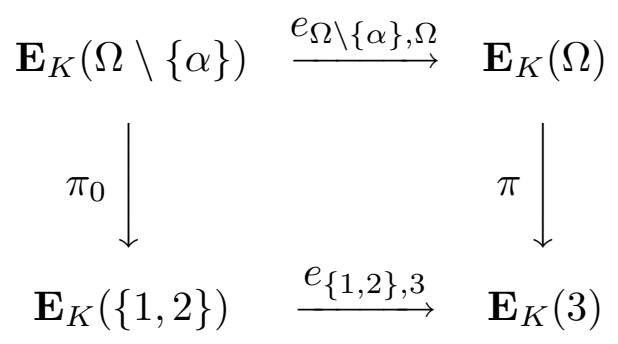


Taking the image under the functor $\mathbf{J}_{K}$ yields that the following diagram commutes:

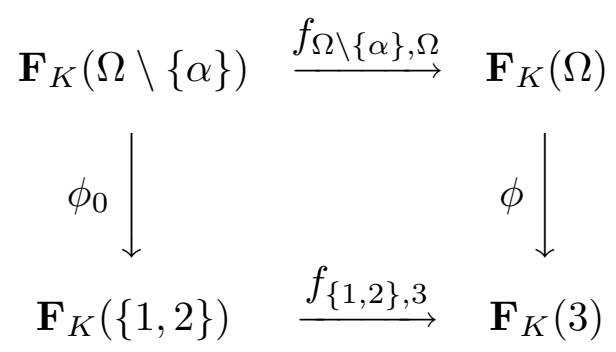

But since $c_{\beta \gamma}$ has support $\Omega \backslash\{\alpha\}$, there exists an element $x$ of $\mathbf{F}_{K}(\Omega \backslash\{\alpha\})$ such that $f_{\Omega \backslash\{\alpha\}, \Omega}(x)=c_{\beta \gamma}$. It follows that $d_{0}=\phi\left(c_{\beta \gamma}\right)=f_{\{1,2\}, 3} \circ \phi_{0}(x)$ belongs to the range of $f_{\{1,2\}, 3}$, hence has support $\{1,2\}$.

- Claim 3.

Now, let $p_{i}, c_{i}, G_{i}(i<3), f$ and $\iota$ be as in Lemmas 2.6 and 2.7 .

Claim 4. Every element of $\mathbf{E}_{K}(3)$ is fixed by $f_{[\iota]}$, and for all $i<3$, we have

$$
(1-2 \varepsilon) c_{i} \leq f_{[\iota]}\left(d_{i}\right) \leq c_{i}+\varepsilon e
$$

Proof of Claim. Since $f_{[\iota]}$ extends $f$, the first part of the statement is trivial. Now let $i<3$. By Claim 3, $3 \backslash\{i\}$ is a support of $d_{i}$, thus, by Lemma 2.7, $c_{i}^{\prime}=f_{[l]}\left(d_{i}\right)$ belongs to $G_{i}$. But by applying $f_{[l]}$ to Claim 2 (ii), we obtain immediately $0, a_{i^{\prime}}+a_{i^{\prime \prime}}-(1+2 \varepsilon) e \leq$ $c_{i}^{\prime} \leq a_{i^{\prime}}+\varepsilon e, a_{i^{\prime \prime}}+\varepsilon e$. But by Lemma 2.6 (i), there exist elements $x_{j}(j<4)$ of $K$ such that $c_{i}^{\prime}=\varphi_{i}\left(\left\langle x_{0}, x_{1}, x_{2}, x_{3}\right\rangle\right)$, and thus, we obtain easily that $0 \leq x_{j} \leq \varepsilon$ for all $j \in\{1,2,3\}$, while $1-2 \varepsilon \leq x_{0} \leq 1+\varepsilon$. The conclusion of Claim 4 follows easily.

- Claim 4.

Now the last part of the proof has come: indeed, by applying $f_{[l]}$ to Claim 2 (i), we obtain, using Claim 4, that $(1-2 \varepsilon)\left(c_{0}+c_{1}\right) \leq a_{2}+2 \varepsilon e+(1+\varepsilon) c_{2}$. However, this is not true! Indeed, let $\lambda$ be any element of $K$ such that $1<\lambda \leq 2$, and let $h: \mathbf{E}_{K}(3) \rightarrow K$ be the unique positive homomorphism sending both $a_{0}$ and $a_{1}$ to $\lambda, a_{2}$ to 1 and $e$ to $2 \lambda$. Thus we have

$$
h \circ p_{0}=h \circ p_{1}=\langle 0,1-\lambda, \lambda, 1\rangle, \quad h \circ p_{2}=\langle 0,0, \lambda, \lambda\rangle,
$$

thus there exists an interpolator $\iota$ on $K$ such that $\iota\left(h \circ p_{0}\right)=\iota\left(h \circ p_{1}\right)=1$ and $\iota\left(h \circ p_{2}\right)=0$ (we need for this the fact that $\lambda \neq 1$, otherwise we would have $h \circ p_{2}=h \circ p_{0}$ ). Therefore, by applying $h_{[\iota]}$ to the inequality $(1-2 \varepsilon)\left(c_{0}+c_{1}\right) \leq a_{2}+2 \varepsilon e+(1+\varepsilon) c_{2}$, one obtains $(1-2 \varepsilon)(1+1) \leq 1+4 \lambda \varepsilon+0$, which can be written $1 \leq(4+4 \lambda) \varepsilon$. This holds for all $\lambda>1$, whence we obtain that $1 \leq 8 \varepsilon$, a contradiction. 
Note that in fact, we could have strengthened Theorem 2.8 by just disproving the existence of elements $c_{\alpha \beta}(\alpha \neq \beta$ in $\Omega$ ) satisfying the inequalities listed in the statement of Claim 1. However, in our opinion, the statement as it is presented here is more "meaningful".

Moreover, it turns out that Theorem 2.8 has many variants, which we did not try to classify. For example, we were able to replace the hypothesis that $\mu$ is order-preserving and the inequality appearing in the hypothesis (b) by the conjunction of the existence of a zero element 0 of $S$ such that $\mu(0)=0$ and the weaker inequality $\mu(x \wedge z)+\mu(y \wedge z) \leq q \cdot \mu(z)+$ $r \cdot \mu(x \wedge y)$ where $q$ and $r$ are elements of $K$ satisfying $0<q<2$ and $0<r<(4 / q)-2$, the (strict) upper bound for $\varepsilon$ depending this time on $q$ and $r$. However, that version does not seem to have immediate K-theoretical consequences, thus we shall not give details about this in this paper. Thus, we shall first harvest some easy (K-theoretical) corollaries of Theorem 2.8, then prove a version of Theorem 2.8 for distributive semilattices (Theorem $2.15)$.

Corollary 2.9. Let $K$ be an ordered field, let $\Omega$ be any set of cardinality at least $\aleph_{2}$. Let $\varepsilon$ be an element of $K$ such that $0 \leq \varepsilon<1 / 8$. Then there exist no lattice $L$ with 1 and no order-preserving map $\mu: L \rightarrow \mathbf{F}_{K}(\Omega)^{+}$satisfying both of the following conditions:

(a) $\mu(1)=e$ and for all $\alpha \in \Omega$, there exists $u_{\alpha} \in L$ such that

$$
a_{\alpha}-\varepsilon e \leq \mu\left(u_{\alpha}\right) \leq a_{\alpha}+\varepsilon e
$$

(b) For all elements $x$ and $y$ of $L$, we have

$$
\mu(x)+\mu(y)=\mu(x \wedge y)+\mu(x \vee y)
$$

Note that condition (b) above is usually expressed by saying that $\mu$ is a valuation on $L$.

Proof. It suffices to prove that $\mu$ satisfies condition (b) of the statement of Theorem 2.8. We proceed:

$$
\begin{aligned}
\mu(x \wedge z)+\mu(y \wedge z) & =\mu(x \wedge y \wedge z)+\mu((x \wedge z) \vee(y \wedge z)) \\
& \leq \mu(x \wedge y \wedge z)+\mu(z) \quad \text { (because } \mu \text { is order-preserving) } \\
& \leq \mu(x \wedge y \wedge z)+\mu(z \vee(x \wedge y)) \quad \text { (same reason) } \\
& =\mu(z)+\mu(x \wedge y) .
\end{aligned}
$$


In particular, the condition of Corollary 2.9 is satisfied when $L$ is a modular complemented lattice (with 0 and 1 ) and $\mu$ is a $\left(\mathbf{F}_{K}(\Omega)^{+}\right.$-valued) measure on $L$ (it is automatically order-preserving because $L$ is sectionally complemented [2]):

Corollary 2.10. Let $K$ be an ordered field, let $\Omega$ be any set of cardinality at least $\aleph_{2}$. Let $\varepsilon$ be an element of $K$ such that $0 \leq \varepsilon<1 / 8$. Then there exist no modular complemented lattice $L$ with 0 and 1 and no measure $\mu: L \rightarrow \mathbf{F}_{K}(\Omega)^{+}$such that $\mu(1)=e$ and for all $\alpha \in \Omega$, there exists $u_{\alpha} \in L$ such that $a_{\alpha}-\varepsilon e \leq \mu\left(u_{\alpha}\right) \leq a_{\alpha}+\varepsilon e$.

Now we can harvest some further corollaries of these results, which allows us to find solutions to several open problems at once. Taking for example $K=\mathbb{Q}$ and $\Omega=\omega_{2}$ in Corollary 2.10, we can immediately settle the question of measurability of all refinement cones (positive cones of interpolation vector spaces are very special cases of refinement cones):

Corollary 2.11. The positive cone of the interpolation vector space $\mathbf{F}_{\mathbb{Q}}\left(\omega_{2}\right)$ is not measurable and has cardinality $\aleph_{2}$.

Corollary 2.10 has also a consequence for the K-theory of von Neumann regular rings:

Corollary 2.12. The positive cone of the interpolation vector space $\mathbf{F}_{\mathbb{Q}}\left(\omega_{2}\right)$ has cardinality $\aleph_{2}$ and is not isomorphic to the monoid $K_{0}(R)^{+}$of stable isomorphism classes of finitely generated projective right $R$-modules for any von Neumann regular ring $R$.

Proof. Denote by $\operatorname{FP}(R)$ the class of all finitely generated projective right $R$-modules, and for all $M \in \mathrm{FP}(R)$, let $[M]$ be the isomorphism class of $M$; denote by $V(R)$ the monoid of all isomorphism classes of elements of $\mathrm{FP}(R)$ (equipped with its algebraic preordering), so that $K_{0}(R)$ is the Grothendieck group of $V(R)$. For all $M \in \operatorname{FP}(R)$, denote by $[M]_{\mathrm{s}}$ the stable isomorphism class of $M$, so that $K_{0}(R)^{+}=\left\{[M]_{\mathrm{s}}: M \in \mathrm{FP}(R)\right\}$. Suppose that $\phi$ is an isomorphism from $K_{0}(R)$ onto $\mathbf{F}_{\mathbb{Q}}\left(\omega_{2}\right)$. There exists $M \in \operatorname{FP}(R)$ such that $\phi\left([M]_{\mathrm{s}}\right)=e$. Since $e$ is an order-unit of $\mathbf{F}_{\mathbb{Q}}\left(\omega_{2}\right)$, there exists a positive integer $n$ such that $\phi\left([R]_{\mathrm{S}}\right) \leq n e$. Furthermore, for all $\alpha<\omega_{2}$, there exists $N_{\alpha} \in \operatorname{FP}(R)$ such that $\phi\left(\left[N_{\alpha}\right]_{\mathrm{s}}\right)=a_{\alpha}$. In particular, $\left[N_{\alpha}\right]_{\mathrm{s}} \leq[M]_{\mathrm{s}}$. Now, we have a problem because since $V(R)$ may not be cancellative, this does not imply a priori that $\left[N_{\alpha}\right] \leq[M]$. But for all $\alpha<\omega_{2}$, there exists a positive integer $n_{\alpha}$ such that $\left[N_{\alpha}\right]+n_{\alpha}[R] \leq[M]+n_{\alpha}[R]$, thus, since $V(R)$ is a refinement monoid [10, Theorem 2.8], it satisfies [24, Lemma 1.11] (which could be called "approximative cancellation property"), thus there exists $N_{\alpha}^{\prime} \in \mathrm{FP}(R)$ such that $\left[N_{\alpha}\right] \leq[M]+\left[N_{\alpha}^{\prime}\right]$ and $9 n n_{\alpha}\left[N_{\alpha}^{\prime}\right] \leq n_{\alpha}[R]$. Again by using the fact that $V(R)$ is a refinement monoid, we obtain elements $\bar{M}_{\alpha}$ and $\bar{N}_{\alpha}$ of $\operatorname{FP}(R)$ such that $\left[N_{\alpha}\right]=\left[\bar{M}_{\alpha}\right]+\left[\bar{N}_{\alpha}\right]$ and $\bar{M}_{\alpha} \subseteq M$ and $\bar{N}_{\alpha} \subseteq N_{\alpha}^{\prime}$. Moreover, since $9 n n_{\alpha}\left[N_{\alpha}^{\prime}\right] \leq n_{\alpha}[R]$, we also have $9 n n_{\alpha}\left[\bar{N}_{\alpha}\right] \leq$ 
$n_{\alpha}[R]$, thus, projecting onto $K_{0}(R)$ and applying $\phi, 9 n n_{\alpha} \phi\left(\left[\bar{N}_{\alpha}\right]_{\mathrm{s}}\right) \leq n_{\alpha} \phi\left([R]_{\mathrm{s}}\right) \leq\left(n n_{\alpha}\right) e$; hence, $\phi\left(\left[\bar{N}_{\alpha}\right]_{\mathrm{s}}\right) \leq(1 / 9) e$, so that $\phi\left(\left[\bar{M}_{\alpha}\right]_{\mathrm{s}}\right) \leq a_{\alpha} \leq \phi\left(\left[\bar{M}_{\alpha}\right]_{\mathrm{s}}\right)+(1 / 9) e$. Now, let $L$ be the (modular, complemented) lattice of all finitely generated submodules of $M$ and let $\mu: L \rightarrow \mathbf{F}_{\mathbb{Q}}\left(\omega_{2}\right)^{+}$defined by the rule $\mu(N)=\phi\left([N]_{\mathrm{S}}\right)$. Then $\mu$ satisfies the hypotheses of Corollary 2.10, a contradiction.

In particular, one cannot have $\mathbf{F}_{\mathbb{Q}}\left(\omega_{2}\right)^{+} \cong V(R)$ for any von Neumann regular ring $R$ (because then, $V(R)$ would be cancellative, thus isomorphic to $K_{0}(R)^{+}$). Note also that Corollary 2.12 implies immediately the corresponding negative result for approximately finite $\mathrm{C}^{*}$-algebras, but that no similar conclusion for general $\mathrm{C}^{*}$-algebras of real rank zero can be drawn by this method: indeed, the finitely generated projective ideals are not necessarily a lattice, while the proof of Theorem 2.8 makes essential use of the meet operation.

To conclude, we shall now prove as promised earlier a version of Theorem 2.8 for semilattices - more specifically, the maximal semilattice quotient of $\mathbf{F}_{K}(\Omega)^{+}$. Unfortunately, the choice of the elements $c_{\alpha \beta}$ of the proof of Theorem 2.8 does not help in this case. However, we will see that this is easy to fix. First, recall the following definition, due to E.T. Schmidt $[\mathbf{2 1}, \mathbf{2 2}]$ :

Definition 2.13. Let $S$ and $T$ be join-semilattices. A homomorphism $\mu: S \rightarrow T$ is weak distributive when for all $x, y$ and $u$ in $S$, if $\mu(x \vee y)=\mu(u)$, then there are $x^{\prime}$ and $y^{\prime}$ in $S$ such that $\mu\left(x^{\prime}\right) \leq \mu(x), \mu\left(y^{\prime}\right) \leq \mu(y)$ and $x^{\prime} \vee y^{\prime}=u$.

The following proposition (first noted by Dobbertin) relates Schmidt's weak distributive homomorphisms with Dobbertin's V-measures:

Proposition 2.14. Let $B$ be a Boolean algebra, let $S$ be a join-semilattice and let $\mu: B \rightarrow S$ be a join-homomorphism. If $\mu$ is a $V$-measure, then $\mu$ is weak distributive.

Proof. Let $x, y$ and $u$ in $B$ such that $\mu(x \vee y)=\mu(u)$. This can be written $\mu(x) \vee \mu(y)=\mu(u)$, whence, since $\mu$ is a V-measure, there are elements $x^{\prime}$ and $y^{\prime}$ of $B$ such that $u=x^{\prime} \oplus y^{\prime}$ (where $\oplus$ means disjoint join) and $\mu\left(x^{\prime}\right)=\mu(x), \mu\left(y^{\prime}\right)=\mu(y)$.

Now, if $G$ is a partially ordered abelian group, we introduce as in [26] two binary relations $\propto$ and $\asymp$ on $G$ by putting

$$
\begin{gathered}
x \propto y \Leftrightarrow(y \geq 0 \text { and }(\exists n \in \mathbb{N})(x \leq n y)), \\
x \asymp y \Leftrightarrow(x \propto y \text { and } y \propto x)
\end{gathered}
$$

Then it is well-known that $\asymp$ is a monoid congruence on $G^{+}$and that $G^{+} / \asymp$ is the maximal semilattice quotient of $G^{+}$, and that furthermore, if $G^{+}$is a refinement monoid 
(i.e., $G$ is an interpolation group), then $G^{+} / \asymp$ is a distributive semilattice (thus also a refinement monoid). For every element $x$ of $G^{+}$, we will denote by $[x]$ the equivalence class of $x$ modulo $\asymp$. We can now state an analogue of Theorem 2.8 for distributive semilattices:

Theorem 2.15. Let $K$ be an ordered field, let $\Omega$ be any set of cardinality at least $\aleph_{2}$. Then there exist no distributive lattice (not necessarily with 0 or 1) $D$ and no weak distributive homomorphism $\mu: D \rightarrow \mathbf{F}_{K}(\Omega)^{+} / \asymp$ with range containing $\left\{\left[a_{\alpha}\right]: \alpha \in \Omega\right\} \cup\left\{\left[b_{\alpha}\right]: \alpha \in \Omega\right\}$.

This implies in particular that $\mathbf{F}_{K}(\Omega)^{+} / \asymp$ is not the range of any weak distributive homomorphism on a distributive lattice, thus not the range of any V-measure on a Boolean algebra. It is in fact Hans Dobbertin who pointed out to us the property of weak distributivity and its connection with the Congruence Lattice Problem $[\mathbf{2 1}, \mathbf{2 2}$, 23], which explains the way we formulate Theorem 2.15.

Proof. The general argument will be much like the one of the proof of Theorem 2.8. For every $\alpha \in \Omega$, there exist by assumption elements $u_{\alpha}^{\prime}$ and $v_{\alpha}^{\prime}$ of $D$ such that $\mu\left(u_{\alpha}^{\prime}\right)=\left[a_{\alpha}\right]$ and $\mu\left(v_{\alpha}^{\prime}\right)=\left[b_{\alpha}\right]$. Thus $\mu\left(u_{\alpha}^{\prime} \vee v_{\alpha}^{\prime}\right)=\left[a_{\alpha}\right]+\left[b_{\alpha}\right]=[e]=\mu(w)$ where we put for example $w=u_{\alpha_{0}}^{\prime} \vee v_{\alpha_{0}}^{\prime}$ for a fixed $\alpha_{0} \in \Omega$. Therefore, by weak distributivity, there exist elements $u_{\alpha}$ and $v_{\alpha}$ of $D$ such that $\mu\left(u_{\alpha}\right) \leq\left[a_{\alpha}\right], \mu\left(v_{\alpha}\right) \leq\left[b_{\alpha}\right]$ and $u_{\alpha} \vee v_{\alpha}=w$. Now, for all $\alpha, \beta$ in $D$, let $c_{\alpha \beta} \in \mathbf{F}_{K}(\Omega)^{+}$such that $\left[c_{\alpha \beta}\right]=\mu\left(u_{\alpha} \wedge v_{\beta}\right)$. Furthermore, for all $\alpha \in \Omega$, let $a_{\alpha}^{\prime}$ and $b_{\alpha}^{\prime}$ in $\mathbf{F}_{K}(\Omega)^{+}$such that $\mu\left(u_{\alpha}\right)=\left[a_{\alpha}^{\prime}\right]$ and $\mu\left(v_{\alpha}\right)=\left[b_{\alpha}^{\prime}\right]$; thus $a_{\alpha}^{\prime} \propto a_{\alpha}$ and $b_{\alpha}^{\prime} \propto b_{\alpha}$, so that we may assume without loss of generality that $a_{\alpha}^{\prime} \leq a_{\alpha}$ and $b_{\alpha}^{\prime} \leq b_{\alpha}$. Moreover, $\left[a_{\alpha}^{\prime}\right]+\left[b_{\alpha}^{\prime}\right]=\mu\left(u_{\alpha} \vee v_{\alpha}\right)=\mu(w)=[e]$, thus there exists $n_{\alpha} \in \mathbb{N}$ such that $e \leq n_{\alpha}\left(a_{\alpha}^{\prime}+b_{\alpha}^{\prime}\right)$. It is the following claim which requires the distributivity of the lattice on which $\mu$ is defined.

Claim 1. For all $\alpha, \beta$ and $\gamma$ in $\Omega$, both of the following conditions hold:

(i) $c_{\alpha \gamma} \propto c_{\alpha \beta}+c_{\beta \gamma}$

(ii) $c_{\alpha \beta} \propto a_{\alpha}, b_{\beta}$;

(iii) $\left(1 / n_{\alpha}\right) e-b_{\alpha} \propto a_{\beta}+c_{\alpha \beta}$.

Proof of Claim. These are simple calculations: for (i), we have, using the fact that $u_{\beta} \vee v_{\beta}=w \geq u_{\alpha}$,

$$
\begin{aligned}
\left(u_{\alpha} \wedge v_{\beta}\right) \vee\left(u_{\beta} \wedge v_{\gamma}\right) & =\left(u_{\alpha} \vee u_{\beta}\right) \wedge\left(u_{\alpha} \vee v_{\gamma}\right) \wedge w \wedge\left(v_{\beta} \vee v_{\gamma}\right) \\
& \geq u_{\alpha} \wedge v_{\gamma}
\end{aligned}
$$

thus, taking the image under $\mu$ of both sides, $\left[c_{\alpha \gamma}\right] \leq\left[c_{\alpha \beta}\right]+\left[c_{\beta \gamma}\right]$. (ii) is immediate. For (iii), $e \leq n_{\alpha}\left(a_{\alpha}^{\prime}+b_{\alpha}^{\prime}\right) \leq n_{\alpha}\left(a_{\alpha}^{\prime}+b_{\alpha}\right)$, whence $\left(1 / n_{\alpha}\right) e-b_{\alpha} \leq a_{\alpha}^{\prime}$. But $u_{\alpha} \leq\left(u_{\alpha} \vee\right.$ $\left.u_{\beta}\right) \wedge w=u_{\beta} \vee\left(u_{\alpha} \wedge v_{\beta}\right)$, whence $\left[a_{\alpha}^{\prime}\right] \leq\left[c_{\alpha \beta}\right]+\left[a_{\beta}^{\prime}\right] \leq\left[c_{\alpha \beta}\right]+\left[a_{\beta}\right]$, which concludes the proof.

Claim 1. 
Then again, for all $\alpha, \beta \in \Omega$, let $F(\{\alpha, \beta\})$ be a finite common support of both $c_{\alpha \beta}$ and $c_{\beta \alpha}$ (the definition of the $c_{\alpha \beta}$ 's is no longer symmetric in $\alpha, \beta$ ); choose again "independent" $\alpha, \beta$ and $\gamma$ and define $\tau, \pi, \phi, G_{i}(i<3)$ as in the proof of Theorem 2.8. Put again $d_{0}=\phi\left(c_{\beta \gamma}\right), d_{1}=\phi\left(c_{\alpha \gamma}\right)$ and $d_{2}=\phi\left(c_{\alpha \beta}\right)$, and $n_{0}=n_{\alpha}, n_{1}=n_{\beta}$ and $n_{2}=n_{\gamma}$.

Claim 2. The following holds (in $\left.\mathbf{F}_{K}(3)\right)$ :

(i) $d_{1} \propto d_{0}+d_{2}$.

(ii) For all $i<3, d_{i} \propto a_{i^{\prime}}, b_{i^{\prime \prime}}$;

(iii) For all $i<3,\left(1 / n_{i^{\prime}}\right) e-b_{i^{\prime}} \propto a_{i^{\prime \prime}}+d_{i}$.

- Claim 2.

Claim 3. For all $i<3,3 \backslash\{i\}$ is a support of $d_{i}$.

- Claim 3.

Claim 4. Every element of $\mathbf{E}_{K}(3)$ is fixed by $f_{[\iota]}$, and for all $i<3$, there exists $\xi_{i} \in K^{+} \backslash\{0\}$ such that $f_{[\iota]}\left(d_{i}\right)=\xi_{i}\left(a_{i^{\prime}}-c_{i}\right)$.

Proof of Claim. By Lemma 2.7 and Claim 3, $f_{[\iota]}\left(d_{i}\right)$ belongs to $G_{i}$, thus, by Lemma 2.6 (i), it can be written $\varphi_{i}(x)$ for some $x \in K^{4}$. By Claim 2 (ii), we obtain that $x \propto$ $\langle 1,1,0,0\rangle,\langle 0,1,0,1\rangle$, thus there exists $\xi_{i} \in K^{+}$such that $x=\left\langle 0, \xi_{i}, 0,0\right\rangle$. Furthermore, by Claim 2 (iii), we have $\left\langle 1 / n_{i^{\prime}}, 1 / n_{i^{\prime}},\left(1 / n_{i^{\prime}}\right)-1,\left(1 / n_{i^{\prime}}\right)-1\right\rangle \propto\left\langle 1, \xi_{i}, 1,0\right\rangle$, thus $\xi_{i}>0$; whence $f_{[\iota]}\left(d_{i}\right)=\xi_{i}\left(a_{i^{\prime}}-c_{i}\right)$.

Claim 4.

Now we can conclude. Indeed, applying $f_{[\iota]}$ to Claim 2 (i) yields a positive integer $n$ such that (using Claim 4)

$$
\xi_{1}\left(a_{0}-c_{1}\right) \leq n\left[\xi_{0}\left(a_{1}-c_{0}\right)+\xi_{2}\left(a_{0}-c_{2}\right)\right]
$$

whence, putting $\lambda=\max \left\{n \xi_{0} / \xi_{1}, n \xi_{2} / \xi_{1}\right\}$, we obtain

$$
a_{0}-c_{1} \leq \lambda\left(a_{1}-c_{0}+a_{0}-c_{2}\right) \text {. }
$$

Now let $h: \mathbf{E}_{K}(3) \rightarrow K$ be the unique positive homomorphism sending $a_{0}$ to $1, a_{1}$ to $2, a_{2}$ to 3 and $e$ to 4 . Note that we have

$$
h \circ p_{0}=\langle 0,1,2,3\rangle, \quad h \circ p_{1}=\langle 0,0,1,3\rangle, \quad h \circ p_{2}=\langle 0,-1,1,2\rangle,
$$

whence there exists an interpolator $\iota$ on $K$ such that $\iota\left(h \circ p_{0}\right)=2, \iota\left(h \circ p_{1}\right)=0$ and $\iota\left(h \circ p_{2}\right)=1$. Therefore, applying $h_{[\iota]}$ to $(*)$ yields $1-0 \leq \lambda \cdot(2-2+1-1)$, i.e., $1 \leq 0$, a contradiction.

In fact, our original proof of non-measurability of $\mathbf{F}_{K}\left(\omega_{2}\right)^{+}$used a similar choice of the $c_{\alpha \beta}$ 's as in the proof above, but these are of no help in the non-distributive case, which 
explains the different choice performed in the proof of Theorem 2.8. In particular, we cannot conclude immediately about a possible extension of Corollary 2.12 (with $V(R)$ instead of $\left.K_{0}(R)\right)$ to the semilattice $\mathbf{F}_{\mathbb{Q}}\left(\omega_{2}\right)^{+} / \asymp$. This leads to the following problem:

Problem 2.16. Is it the case that for any distributive (join-) semilattice $S$ with 0 and 1 , there exists a von Neumann regular ring $R$ such that $(V(R),[R]) \cong(S, 1)$ ?

As Corollary 2.12 shows, the analogue of this problem for positive cones of interpolation vector spaces over the rationals has a negative answer; but we have seen that distributivity of the lattice is used in an essential way in the proof of Theorem 2.15, which makes more likely the possibility of a positive answer to Problem 2.16. But one has to be warned that such a positive answer would probably be very hard to obtain, for the reason that its conclusion implies that the semilattice of all compact congruences of the lattice $\mathcal{L}\left(R^{2}\right)$ of finitely generated submodules of $R^{2}$ is also isomorphic to $S$, thus solving positively the Congruence Lattice Problem!

A natural lattice-theoretical weakening of Problem 2.16 is to ask whether for every distributive semilattice $S$ (with 0 and 1 ), there exist a modular complemented lattice $L$ and a normalized perspectivity-invariant $\mathbf{V}$-measure $\mu: L \rightarrow S$, the concept of Vmeasure being defined as for Boolean algebras and perspectivity invariance meaning that $a \sim b$ (i.e., the existence of $c$ such that $a \oplus c=b \oplus c$ ) implies that $\mu(a)=\mu(b)$ (this is of course trivial for Boolean algebras). The bridge between both problems would then be the following (probably very difficult)

Problem 2.17. Let $(M, u)$ be a conical refinement monoid with order-unit. Prove that there exists a von Neumann regular ring $R$ such that $(V(R),[R]) \cong(M, u)$ if and only if there exists a perspectivity-invariant normalized $\mathrm{V}$-measure $\mu: L \rightarrow M$ for some complemented modular lattice $L$.

Of course, the "measure-theoretical" condition is necessary; moreover, it is realized for $|M| \leq \aleph_{1}$ by Dobbertin's results (while the $V(R)$ counterpart is the fundamental open problem stated in [12]). A positive evidence for this problem is of course von Neumann's Coordinatization Theorem [20]. The latter, plus some additional work, also imply that Problem $2.1^{\text {r7 }}$ can be conveniently stated in a purely lattice-theoretical way: indeed, it can be shown that for any regular ring $R$, isomorphism of two elements of the lattice $\mathcal{L}\left(R^{4}\right)$ is equivalent to "projectivity by decomposition" ( $a$ and $b$ are said to be projective by decomposition when there are decompositions $a=\oplus_{i<n} a_{i}$ and $b=\oplus_{i<n} b_{i}$ such that $a_{i}$ is projective to $b_{i}$ for all $\left.i<n\right)$.

Note also that it can be shown, mimicking the proof of [3, Theorem 4.1] (see also [1, Proposition 1.1]), that if $L$ is any modular sectionally complemented lattice, if $M$ is a 
commutative monoid and if $\mu: L \rightarrow M$ is a perspectivity-invariant $\mathrm{V}$-measure as defined above, then $M$ is conical and the range of $\mu$ is a lower subset of $M$ (for the algebraic preordering) satisfying the refinement property (in the sense that any equality of the form $c=a_{0}+a_{1}=b_{0}+b_{1}$ where $c$ belongs to $\mu[L]$ admits a refinement). In particular, in the semilattice context, the existence of $\mu$ as above forces the semilattice to be distributive. These two final remarks, plus the main results of this paper, seem to suggest the possibility of an answer to Problem 2.17 via measures on complemented, modular lattices.

Note added. In the meantime, the author has answered negatively Problem 2.16. In fact, when $S$ is the semilattice counterexample of Theorem 2.15, there is no sectionally complemented lattice $L$ whose semilattice of compact congruences is isomorphic to $S$.

\section{BIBLIOGRAPHY}

[1] P. ARA, K.R. GOODEARL, E. PARDO and K.C. O’MEARA, "Separative cancellation for projective modules over exchange rings", preprint.

[2] G. BIRKHOFF, "Lattice theory", American Mathematical Society Colloquium Publications, vol. 25, 3rd edition, seventh printing, 1993.

[3] P. CRAWLEY and B. JÓNSSON, "Refinements for infinite direct decompositions of algebraic systems", Pacific Journal of Mathematics 14 (1964), pp. 797-855.

[4] H. DOBBERTIN, "On Vaught's criterion for isomorphism of countable Boolean algebras", Algebra Universalis 15 (1982), pp. 95-114.

[5] H. DOBBERTIN, "Refinement monoids, Vaught Monoids, and Boolean Algebras", $M a-$ thematische Annalen 265 (1983), pp. 475-487.

[6] H. DOBBERTIN, "Measurable refinement monoids and applications to distributive semilattices, Heyting algebras and Stone spaces", Mathematische Zeitschrift 187 (1984), pp. $13-21$.

[7] H. DOBBERTIN, "Vaught's measures and their applications in lattice theory", Journal of Pure and Applied Algebra 43, No. 1 (1986), pp. 27-51.

[8] E.G. EFFROS, D.E. HANDELMAN and C-L. SHEN, "Dimension groups and their affine representations", American Journal of Mathematics, vol. 102 (1980), No. 2, pp. $385-407$.

[9] G.A. ELLIOTT, "On the classification of inductive limits of sequences of semisimple finite-dimensional algebras", Journal of Algebra 38 (1976), pp. 29-44.

[10] K.R. GOODEARL, "Von Neumann Regular Rings", Pitman, London, 1979; Second Ed., Krieger, Malabar, Fl., 1991. 
[11] K.R. GOODEARL, "Partially ordered abelian groups with the interpolation property", Mathematical surveys and monographs, number 20, American Mathematical Society, 1986.

[12] K.R. GOODEARL, "Von Neumann regular rings and direct sum decomposition problems", Abelian Groups and Modules, Padova 1994 (A. Facchini and C. Menini, eds.), Dordrecht (1995) Kluwer, pp. 249-255.

[13] K.R. GOODEARL and D.E. HANDELMAN, "Tensor products of dimension groups and $K_{0}$ of unit-regular rings", Canadian Journal of Mathematics, vol. 38, No. 3 (1986), pp. 633-658.

[14] G. GRÄTZER, "General Lattice Theory”, Birkhäuser Verlag, Basel 1978.

[15] P.A. GRILLET, "Interpolation properties and tensor products of semigroups", Semigroup Forum 1 (1970), pp. 162-168.

[16] A.P. HUHN, "On the representation of algebraic distributive lattices III", Acta Scientiarum Mathematicarum (Szeged) 53 (1989), pp. 11-18.

[17] J. KETONEN, "The structure of countable Boolean algebras", Annals of Mathematics 108, No. 1 (1978), pp. 41-89.

[18] C. KURATOWSKI, "Sur une caractérisation des alephs", Fundamenta Mathematic 38 (1951), pp. 14-17.

[19] D. MYERS, "Lindenbaum-Tarski Algebras", in "Handbook of Boolean Algebras", vol. 3, pp. 1167-1196, edited by J.D. Monk with R. Bonnet, Elsevier, Amsterdam, 1989.

[20] J. von NEUMANN, "Continuous geometry", Princeton University Press, Princeton, New Jersey, 1960.

[21] E.T. SCHMIDT, "Kongruenzrelationen algebraischer Strukturen", VEB Deutscher Verlag der Wissenschaften, Berlin, 1969.

[22] E.T. SCHMIDT, "The ideal lattice of a distributive lattice with 0 is the congruence lattice of a lattice", Acta Scientiarum mathematicarum (Szeged) 43 (1981), pp. 153168.

[23] M. TISCHENDORF, "On the representation of distributive semilattices", Algebra Universalis 31 (1994), pp. 446-455.

[24] F. WEHRUNG, "Injective positively ordered monoids I", Journal of Pure and Applied Algebra 83 (1992), pp. 43-82.

[25] F. WEHRUNG, "Restricted injectivity, transfer property and decompositions of separative positively ordered monoids", Communications in Algebra 22 (5) (1994), pp. $1747-1781$. 
[26] F. WEHRUNG, "Embedding simple commutative monoids into simple refinement monoids", to appear in Semigroup Forum. 